Heike Trappe

\title{
Väterzeit - das Elterngeld als Beschleuniger von Gleichstellung?
}

\author{
Time with fathers - the parental leave benefit as promoter of gender \\ equity?
}

\begin{abstract}
Zusammenfassung:
Im Zentrum des vorliegenden Beitrags steht der Elterngeldbezug von Vätern im Kontext der Partnerschaft in den Bundesländern Bayern, Mecklenburg-Vorpommern und Schleswig-Holstein. Grundlage der Analysen sind von den Elterngeldstellen erhobene Daten für zwischen 2007 und 2009 geborene Kinder. Es wird danach gefragt, welche Paare überhaupt einen Partnerantrag stellen und wodurch sich Paare mit unterschiedlicher Dauer des Elterngeldbezugs durch Väter voneinander unterscheiden. Die Entscheidung für einen Partnerantrag folgt im Wesentlichen aus ökonomischen Theorien abgeleiteten Erwartungen, denn eine Erwerbstätigkeit und ein hohes Einkommen der Frau begünstigen diese. Väter, bei denen der Elterngeldbezug mit einer über die „Bonusmonate" hinausgehenden Elternzeit einhergeht, sind eine sehr selektive Gruppe, die im Zeitverlauf sogar kleiner geworden ist. Ein nichteheliches Zusammenleben, das Leben in einer Großstadt, eine höhere Anzahl von Kindern sowie eine spezifische Lebenssituation der Partnerin (z.B. selbstständige Tätigkeit, Beendigung einer Ausbildung) wirken sich positiv auf eine längere Elternzeit des Vaters aus.
\end{abstract}

Schlagwörter: Eltern, Elterngeld, Elternzeit, Vaterschaft, ökonomische Ressourcen.

\begin{abstract}
:
This contribution centres on men who claim parental leave benefits in the federal states of Bavaria, Mecklenburg-Western Pomerania and Schleswig-Holstein. The analyses are based on register data collected by local authorities for children born between 2007 and 2009. First, it is asked about the determinants underlying the take-up of fathers' parental leave benefits. Then, couples with different durations of fathers' parental leave are investigated more closely. Fathers' decision to take any parental leave follows essentially economic considerations within the couple. Female partners' employment and their relative earnings have a positive impact. Couples where fathers' parental leave exceeds the "daddy quota" represent quite a select group that has become smaller over time. Living in a non-marital union or in a large city, having more children as well as female partners' particular situation (e.g., self-employment, in education) facilitate a longer parental leave of fathers.
\end{abstract}

Key words: parents, parental leave benefit; parental leave, fatherhood, economic resources. 


\section{Einleitung}

Sozialwissenschaftliche Studien, die den Wandel der Vaterrolle in Deutschland thematisieren, kommen - je nach Datengrundlage und Ausrichtung - zu deutlich unterschiedlichen Einschätzungen. Während beispielsweise die Trendstudie „Moderne Väter“ einen spürbaren Wertewandel ausmacht, der eine aktive Vaterschaft zunehmend unterstützt (Väter gGmbH 2012), kommt Schneider (2010) zu einer eher verhaltenen Bilanz: „Der Wandel vom Ernährer zum Erzieher ist eine Idee, die in der Praxis noch kaum angekommen ist und der sogenannte ,Neue Vater' lässt sich empirisch nicht entdecken ... Der Anstieg der Inanspruchnahme der sogenannten Vätermonate ... kann hier als Einstieg in einen allmählichen Wandel gedeutet werden. Anlass für eine bereits erfolgte Trendwende geben die bisherigen Zahlen jedoch nicht" (Schneider 2010: 14).

Vor diesem Hintergrund geht es in dem vorliegenden Beitrag um Merkmale der Partnerschaft und regional variierende Opportunitätsstrukturen, welche den Elterngeldbezug durch Väter erleichtern. Gefragt wird zum einen danach bei welchen Paaren überhaupt ein Partnerantrag gestellt wird. Schwerpunktmäßig werden jedoch die Paare miteinander verglichen, in denen Väter bis zu zwei Monate oder über einen darüber hinausgehenden Zeitraum Elterngeld beanspruchen. Handelt es sich insbesondere bei letzterer Gruppe um eine Avantgarde der Geschlechtergleichstellung? Zur Beantwortung dieser Fragen werden einzigartige Daten genutzt, welche durch die Elterngeldstellen bei der Beantragung des Elterngeldes für zwischen 2007 und 2009 geborene Kinder erhoben wurden. Diese stehen für drei ausgewählte Bundesländer (Bayern, Mecklenburg-Vorpommern, SchleswigHolstein) vollständig zur Verfügung und bieten damit sowohl Ansatzpunkte für die Untersuchung von Ost-/Westunterschieden, aber auch von Stadt-/Landunterschieden. Zusätzlich werden Daten der Allgemeinen Bevölkerungsumfrage der Sozialwissenschaften (ALLBUS) herangezogen, um für die drei Bundesländer Einstellungen von Männern zur Rolle der Frau und den Konsequenzen der Müttererwerbstätigkeit berücksichtigen zu können.

Im folgenden Abschnitt wird sowohl auf Basis der Elterngeldstatistik als auch vorliegender sozialwissenschaftlicher Untersuchungen zum Elterngeldbezug von Vätern der Forschungsstand zusammengefasst, bevor knapp auf zugrundeliegende theoretische Vorstellungen und daraus abgeleitete Hypothesen rekurriert wird. Der Beschreibung der verwendeten Daten, der Analysestrategie und der Methoden folgt eine Darstellung der deskriptiv und multivariat ermittelten Ergebnisse zum väterlichen Elterngeldbezug und dessen Dauer. Abgeschlossen wird der Beitrag durch eine Diskussion der Ergebnisse und einige Überlegungen zu möglichen Auswirkungen des Elterngeldes auf die Gleichstellung von Frauen und Männern. 


\section{Forschungsstand und Theorie}

\subsection{Amtliche Statistik zum Elterngeld}

Die im Kontext der Elterngeldreform deutlich gestiegene Inanspruchnahme der Elternzeit durch Väter wird seitens politischer Entscheidungsträger immer wieder als Erfolg vermeldet (BMFSFJ 2012a, 2012b). Der Anteil der Väter, die unter der alten Regelung Erziehungsgeld erhielten, schwankte in den Jahren vor 2007 zwischen ein und drei Prozent (Geisler/Kreyenfeld 2011). Für im Jahr 2007 geborene Kinder wurde von 18 Prozent der Väter Elterngeld bezogen. Dieser Anteil erhöhte sich in den Folgejahren kontinuierlich und betrug für im Jahr 2011 geborene Kinder 27 Prozent, d.h. für jedes vierte Kind wurde auch durch den Vater Elterngeld bezogen. Allerdings gibt es deutliche regionale Unterschiede: An der Spitze der Väterbeteiligung lagen die Bundesländer Bayern, Sachsen und Berlin, während das Saarland und Bremen am unteren Ende rangierten. Die Mütterbeteiligung wird mit konstant 96 Prozent ausgewiesen (Statistisches Bundesamt 2012a, 2013).

Bezüglich der Dauer des Elterngeldbezugs weist die amtliche Statistik kaum Veränderungen im Zeitverlauf aus: Drei von vier Vätern beziehen Elterngeld für maximal zwei Monate, während neun von zehn Müttern dies für mindestens 12 Monate tun (Statistisches Bundesamt 2012a). Ein genauerer Blick in die Elterngeldstatistik zeigt jedoch bereits, dass sich die durchschnittliche Dauer des Elterngeldbezugs bei Vätern im Verlauf der Zeit verringert hat. Für im Jahr 2008 geborene Kinder betrug diese 3,9 Monate und für im Jahr 2011 geborene Kinder nur noch 3,3 Monate. Dementsprechend hat der Anteil der Väter mit einem maximal zweimonatigen Elterngeldbezug zugenommen. Im Unterschied zu Müttern mit Elterngeldbezug variiert die durchschnittliche Dauer bei Vätern systematisch mit dem Erwerbsstatus vor der Geburt des Kindes. Vor allem nicht erwerbstätige Väter beziehen Elterngeld für einen durchschnittlich längeren Zeitraum (Statistisches Bundesamt 2012b, 2013). Doch welche Charakteristika weisen Paare auf, in denen die Väter Elterngeld beziehen? Welche Rolle spielen ökonomische Abwägungen innerhalb der Partnerschaft für ihre Entscheidung, die Erwerbstätigkeit zeitweilig einzuschränken und Elterngeld zu beziehen? Sind diese Überlegungen gleichermaßen relevant, wenn es um die Aufteilung der Dauer des Elterngeldbezugs innerhalb der Partnerschaft geht? Gibt es bereits Hinweise darauf, dass das Elterngeld Auswirkungen auf die Gleichstellung der Geschlechter hat?

\subsection{Sozialwissenschaftliche Untersuchungen}

Auch wenn die Befundlage zu einflussreichen Determinanten der Väterbeteiligung seit der Elterngeldreform noch vorläufig ist, beginnt sich ein Bild abzuzeichnen, nach dem wesentliche soziodemographische Einflussfaktoren ein höheres Alter des Vaters sowie ein Leben in Großstädten sind (Pfahl/Reuyß 2010; Reich 2011; RWI Essen 2008, 2009). Hinzu kommen Merkmale des Beschäftigungsverhältnisses, die einen Elterngeldbezug des Vaters erleichtern, wie eine Tätigkeit im öffentlichen Dienst, in größeren Unternehmen und ein unbefristeter Arbeitsvertrag (Geisler/Kreyenfeld 2012; Pfahl/Reuyß 2010; Reich 2011). Hinsichtlich zugrundeliegender Motivationen von Vätern zur Nutzung von Eltern- 
geld und Elternzeit gibt insbesondere die qualitative Studie von Richter (2012) Aufschluss, die auf einer inhaltsanalytischen Auswertung von Paarinterviews basiert. Zentral ist demnach der Wunsch, die gesamte Familie zu entlasten, eine enge Beziehung zum Kind zu entwickeln, den beruflichen Wiedereinstieg der Partnerin zu erleichtern, sich eine berufliche Auszeit zu gönnen oder innerhalb der Partnerschaft Erwerbs- und Familienarbeit möglichst paritätisch zu verteilen. Die Einführung des Elterngeldes und der Partnermonate war in dieser Studie für alle interviewten Paare eine ganz wesentliche Rahmenbedingung für ihre Entscheidung, denn damit wurde die Verhandlungsbasis des Vaters in zweifacher Hinsicht gestärkt, gegenüber der Partnerin und gegenüber dem Arbeitgeber (Richter 2012: 105ff.). Bezüglich der Wirkung ökonomischer Ressourcen sind die Ergebnisse vorliegender Studien am wenigsten eindeutig. Geisler und Kreyenfeld (2012) folgern, dass vor allem hoch qualifizierte Väter und Männer, die eine höhere Qualifikation als ihre Partnerin haben, von der Reform profitiert haben. Da sie nicht für das Einkommen kontrollieren, ist dieses Ergebnis sowohl mit einem verstärkten ökonomischen Anreiz des Elterngeldes für diese Gruppen von Männern als auch mit einer normativen Interpretation kompatibel. Reich hingegen findet Hinweise auf einen U-förmigen Zusammenhang von Bildung und Einkommen zur väterlichen Inanspruchnahme von Elternzeit, d.h. eine höhere Konzentration im oberen und unteren Bereich der Bildungs- und Einkommensverteilung. Sie zeigt auch, dass von der Erwerbstätigkeit und vom Einkommen der Partnerin eine positive Wirkung ausgeht, was mit ressourcentheoretischen Überlegungen im Einklang steht (vgl. auch Pull/Voigt 2010). Ausgehend von den noch unklaren Forschungsbefunden zur Wirkung ökonomisch verwertbarer Ressourcen im Hinblick auf den Elterngeldbezug von Vätern wird im vorliegenden Beitrag eine differenzierte Einbeziehung verschiedener Dimensionen (Erwerbsstatus, Einkommen) für beide Partner als unerlässlich angesehen.

In Deutschland wurde bislang ausschließlich untersucht, welche Bedingungsfaktoren den Elterngeldbezug durch Väter begünstigen, während die - unter Gleichstellungsaspekten besonders relevante - Dauer der von ihnen genutzten Elternzeit nicht analysiert wurde (vgl. für eine Ausnahme Trappe 2013). Ein Blick auf den internationalen Forschungsstand, der sich vor allem auf den skandinavischen Raum bezieht ${ }^{1}$, offenbart, dass hier die Dauer der Elternzeit von Vätern im Zentrum des Interesses steht. Grundlegend dafür ist, dass die skandinavischen Länder überwiegend über zuverlässige Registerdaten verfügen, welche eine umfassende Analyse der Väterbeteiligung an der Elternzeit erst ermöglichen. Umfangreichere ökonomische Ressourcen beider Partner begünstigen demnach eine längere Elternzeit des Vaters (für Schweden: Bygren/Duvander 2006; Duvander/Johansson; Sundström/Duvander 2002, für Norwegen: Lappegard 2008; Naz 2010). Mit dem vorliegenden Beitrag wird auch ein Anschluss an die internationale Forschung angestrebt, indem nicht nur die Beteiligung, sondern auch die Dauer der durch Väter genutzten Elternzeit analysiert wird.

1 In den skandinavischen Ländern wurde bereits seit den frühen 1990er Jahren im Kontext großzügig ausgestalteter Erziehungszeitregelungen eine explizit an Väter adressierte Politik verfolgt (für einen ausgezeichneten Überblick: Haas/Rostgaard 2011). Ein interessanter Unterschied zur Regelung über die „Vätermonate“ in Deutschland besteht darin, dass diese beispielsweise in Schweden nicht zeitgleich zur Elternzeit der Mutter genommen werden dürfen, sondern exklusiv dem Vater vorbehalten sind (Duvander/Johansson 2012). 


\subsection{Theoretischer Hintergrund und Hypothesen}

Die mit der Elterngeldreform verbundene Umstellung von einer pauschalen Transfer- auf eine Lohnersatzleistung, die sich an der Höhe des zuvor durchschnittlich erzielten Nettoeinkommens orientiert, setzt klare Anreize für ökonomisch motivierte Entscheidungen innerhalb der Partnerschaft. Gleiches gilt für die Tatsache, dass die Dauer des Elterngeldbezugs nur dann vollständig ausgeschöpft werden kann, wenn ein Partner mindestens zwei Monate beansprucht, die Gesamtdauer also innerhalb der Partnerschaft aufgeteilt wird. ${ }^{2}$ Mit dieser explizit an Väter adressierten Regelung rückt diese Gruppe deutlich stärker als zuvor ins Blickfeld der Familienpolitik (Baronsky et al. 2012). Unter Berücksichtigung dieser Rahmenbedingungen sind Paare im Vorfeld ihrer Entscheidungen dazu in der Lage, den voraussichtlichen Einkommensausfall zu kalkulieren. Insofern bieten sich ökonomische Erklärungen an, auch wenn bedacht wird, dass im Zuge der Familiengründung oder -erweiterung der finanzielle Bedarf für deren Unterhalt steigt. Da eine Gleichstellungsperspektive auf mögliche Wirkungen des Elterngeldes ein Nachdenken über veränderte Geschlechterrollen erfordert, werden die ökonomischen Theorien zur Arbeitsteilung durch den soziologischen Doing-Gender-Ansatz ergänzt. Da es sich bei diesen Theorien um breit rezipierte und wohlbekannte Ansätze handelt, sollen hier nur deren Kernaussagen und die daraus abgeleiteten Hypothesen dargestellt werden.

Ausgehend von der neuen Haushaltsökonomie (Becker 1991; Berk/Berk 1983) wird angenommen, dass beide Partner von einer Spezialisierung profitieren, welche die jeweiligen komparativen Vorteile im Bereich bezahlter Erwerbsarbeit und unbezahlter Reproduktionsarbeit ausnutzt. Eine Reduzierung der Erwerbsarbeit geht mit Opportunitätskosten einher, die sich aus dem Einkommensausfall, reduzierten Karrierechancen und einer möglichen Entwertung erworbener Qualifikationen ergeben. Dementsprechend sollten Väter vor allem dann für einen längeren Zeitraum Elterngeld beziehen, wenn sie bereits vor der Geburt des Kindes nicht oder in deutlich geringerem Umfang als ihre Partnerin erwerbstätig waren.

Die Verhandlungs- bzw. Ressourcentheorie knüpft unmittelbar an den Macht- und Aushandlungsaspekt innerhalb von Partnerschaften an (Blood/Wolfe 1960; Ott 1992). Demnach streben beide Partner eine Maximierung ihres individuellen Nutzens an und setzen ihre jeweiligen Ressourcen, wie Bildung, Beruf und Einkommen in innerpartnerschaftlichen Aushandlungen ein (Lundberg/Pollak 1996). Der Partner mit den besseren Chancen für eine Verwertung der Ressourcen auf dem Arbeitsmarkt ist in der günstigeren Verhandlungsposition, um die unbezahlte Arbeit an den jeweils anderen zu verweisen oder an Dritte zu delegieren (Mannino/Deutsch 2007; Naz 2010). Eine - verglichen mit der Partnerin - ungünstigere ökonomische Situation des Mannes (z.B. Erwerbsstatus, Einkommen), geht mit einer schwächeren Verhandlungsposition einher und macht deshalb seinen längeren Elterngeldbezug wahrscheinlicher. Eine günstigere ökonomische Ressourcenausstattung des Mannes sollte einen entgegengesetzten Effekt haben. Da der Einkommensersatz durch das Elterngeld lediglich etwa zwei Drittel beträgt, sollte die Höhe

2 Auch Alleinerziehende erhalten das Elterngeld für 14 Monate. Bei einer Analyse auf der Paarebene wird diese überwiegend aus Frauen bestehende Gruppe jedoch ausgeschlossen. 
des vor der Geburt erzielten gemeinsamen Einkommens die Entscheidung des Vaters für einen längeren Elterngeldbezug positiv beeinflussen.

Der soziologisch orientierte Doing-Gender-Ansatz (West/Zimmermann 1987) betont die soziale Konstruiertheit des Geschlechts und besagt, dass Geschlechtsidentitäten im Alltag ständig reproduziert werden müssen, um wirksam sein zu können. Gerade in Bezug auf Elternschaft und die Partizipation von Vätern an der Kinderbetreuung ist davon auszugehen, dass es nicht allein um rationale Abwägungen, sondern auch um identitätsstiftende Aufgabenbereiche geht, die internalisierten Überzeugungen von Weiblichkeit und Männlichkeit folgen (Coltrane 2009). Der Doing-Gender-Ansatz ermöglicht es zu verstehen, dass Frauen und Männer entsprechend ihrem internalisierten Verständnis von Geschlechterrollen eine Erwerbsunterbrechung von Vätern für die Betreuung ihrer kleinen Kinder gutheißen oder dies eher ablehnen. Davon ausgehend kann auch verdeutlicht werden, dass Frauen möglicherweise eine längere Elternzeit ihres Partners nicht unterstützen, wenn sie dafür selbst ihre Zeit mit dem Kind reduzieren müssten (im Sinne eines gatekeeping durch die Mutter). Dieser Aspekt ist wichtig, denn er unterstreicht, dass ein (längerer) Elterngeldbezug durch den Vater nicht nur von ihm selbst abhängt, sondern auch durch die Partnerin aktiv unterstützt werden muss. Es wird angenommen, dass in einer Situation, in der beide Partner über vergleichbare ökonomisch verwertbare Ressourcen verfügen oder das Paar über ein hohes gemeinsames Einkommen verfügt am ehesten ein Spielraum existiert, um internalisierten Geschlechtsidentitäten zu folgen. Ein positiver Effekt einer derartigen Situation auf einen längeren Elterngeldbezug des Mannes legt die Relevanz entsprechender Einstellungen zur Vaterschaft nahe. Auch sollten sich egalitäre Vorstellungen von Geschlechterrollen stärker in der Dauer des Elterngeldbezugs als in der Beteiligung widerspiegeln.

\section{Daten, Analysestrategie und Methode}

\subsection{Verwendete Datensätze und Analysestrategie}

Die empirischen Analysen zum Partnerantrag und zur Aufteilung der Bezugsmonate innerhalb der Partnerschaft stützen sich auf einzigartige Daten, die im Zusammenhang mit der Beantragung des Elterngeldes durch die Elterngeldstellen erhoben wurden. ${ }^{3}$ Es handelt sich dabei um vollständige Angaben für alle Geburten, die zwischen 2007 und 2009 in den Bundesländern Bayern, Mecklenburg-Vorpommern und Schleswig-Holstein erfolgten und für die ein Antrag auf den Bezug von Elterngeld gestellt wurde. Enthalten sind Informationen der Antragsteller zum Familienstand, zum Alter, zum Erwerbsstatus ein Jahr vor der Geburt des Kindes und zur Anzahl der Kinder im Haushalt. Außerdem sind die genauen Elterngeldbeträge sowie die Anzahl der Bezugsmonate verzeichnet. Die exakten Elterngeldbeträge lassen in Kombination mit dem Erwerbsstatus Rückschlüsse

3 Mein ausdrücklicher Dank für die Unterstützung bei der Bereitstellung der Daten gilt an dieser Stelle Dr. Heiko Will vom Landesamt für Gesundheit und Soziales Mecklenburg-Vorpommern, Wolfgang Rätsch vom Landesamt für soziale Dienste Schleswig-Holstein und Erwin Manger vom Zentrum Bayern Familie und Soziales. 
auf das vorherige durchschnittliche Nettoerwerbseinkommen mit sehr geringen Anteilen fehlender Werte zu. ${ }^{4}$ Da es sich um das durch eine Erwerbstätigkeit erzielte Nettoeinkommen handelt, wird dessen Höhe u.a. auch durch die Wahl der Steuerklasse beeinflusst. Doch bildet genau dieses Nettoeinkommen eine wesentliche Bedingung für die Aushandlung des Elterngeldbezugs innerhalb der Partnerschaft. Insofern ist klar, dass die in der Partnerschaft stattfindenden Aushandlungsprozesse vor dem Hintergrund sämtlicher relevanter Rahmenbedingungen, wie eines möglichen Zusammenwirkens des Ehegattensplittings mit dem Elterngeld, erfolgen (Beblo 2012). Die genauen Elterngeldangaben sowie die Möglichkeit, die Aufteilung der Bezugsmonate innerhalb des Elternpaares rekonstruieren zu können, verdeutlichen das große Potential der Daten. Ihr Nachteil besteht darin, dass nur dann Informationen zu beiden Partnern vorliegen, wenn diese tatsächlich Elterngeld beantragt haben. Insofern ist es nicht - wie in skandinavischen Ländern - möglich, auch die Mütter und Väter zu berücksichtigen, die keine Elternzeit bzw. kein Elterngeld beanspruchen.

Im ersten Schritt erfolgen daher Analysen zum Elterngeldbezug durch einen Partner. ${ }^{5}$ Diese Analysen stützen sich auf alle Geburten zwischen 2007 und 2009, für die ein Elterngeldantrag durch die Mutter gestellt wurde. Sie können jedoch, sofern kein Partnerantrag gestellt wurde, nur die Angaben der Mutter berücksichtigen. Im zweiten und zentralen Schritt steht die Dauer des Elterngeldbezugs durch den Partner im Zentrum des Interesses. Die auschlaggebende Idee ist, dass die Dauer des Elterngeldbezugs durch Väter sehr viel stärker die Arbeitsteilung bei der Kleinkindbetreuung widerspiegelt als die bloße Väterbeteiligung. Hier wird zunächst geprüft, wie stark die Dauer des Elterngeldbezugs mit dem Erwerbsstatus des Vaters vor der Geburt des Kindes variiert. Alle weiteren Analysen konzentrieren sich dann auf Frauen mit vor der Geburt des Kindes erwerbstätigen Partnern bzw. auf Doppelverdienerpaare. Mit dieser Selektion des Samples soll sichergestellt werden, dass ein Elterngeldbezug auch mit einer tatsächlichen Unterbrechung oder Reduzierung der Erwerbstätigkeit des Vaters im Sinne einer Elternzeit verbunden ist. ${ }^{6}$ Insbesondere bei Doppelverdienerpaaren liegt der Fokus auf denjenigen, welche in einer Situation sind, die Gesamtdauer des Elterngeldbezugs auch miteinander aushandeln zu können. In einem dritten und letzten Schritt werden die für die drei Bundesländer Bayern, Mecklenburg-Vorpommern und Schleswig-Holstein ermittelten Ergebnisse zur Dauer des Elterngeldbezugs von Vätern und den zugrundeliegenden Determinanten in Beziehung zu

4 In den vorliegenden Daten sind im Falle einer Zahlung des Elterngeldes in Höhe des Mindest- oder Höchstsatzes (300 bzw. 1.800 Euro) auch immer die exakten Angaben ausgewiesen, welche sich aus dem Erwerbseinkommen ergeben würden. Im Falle einer Teilzeittätigkeit während des Elterngeldbezugs ist neben dem Teilelterngeld, welches die Person bezogen hat, auch der Betrag verzeichnet, den sie bekommen hätte, wenn sie nicht erwerbstätig gewesen wäre. Insofern ist auch in diesen Fällen eine valide Rekonstruktion des durchschnittlichen, vor der Geburt des Kindes erzielten Nettoeinkommens möglich.

5 In einigen Fällen handelt es sich hier auch um eine Partnerin (z.B. bei eingetragenen Lebenspartnerschaften). Diese wurden hier noch nicht ausgeschlossen, da sie eine interessante zusätzliche Dimension eröffnen.

6 Nur für die vor der Geburt des Kindes erwerbstätigen Väter impliziert ein Elterngeldbezug auch eine Einschränkung ihrer Erwerbstätigkeit und damit eine Veränderung ihres Alltags und der ökonomischen Situation ihrer Familie. Beides trifft auf zuvor nicht erwerbstätige Väter, die Elterngeld beziehen, nur eingeschränkt zu. 
auf Basis des ALLBUS bestimmten Einstellungsdimensionen gesetzt und somit kontextuell eingebettet.

\subsection{Operationalisierung der Variablen}

Für die Untersuchung der Inanspruchnahme des Elterngeldes wurde für alle im Beobachtungszeitraum geborenen Kinder danach unterschieden, ob ein Partnerantrag vorliegt oder nicht. Bezogen auf die Aufteilung der Bezugsdauer wurde ebenfalls eine Variable mit zwei Ausprägungen („Partner bezieht für ein oder zwei Monate Elterngeld“ bzw. „Partner bezieht für mehr als zwei Monate Elterngeld“) gebildet, um die Gruppe der Väter genauer zu analysieren, welche über die „Vätermonate“ hinaus Elterngeld beziehen. ${ }^{7}$

Als Kontrollvariablen gehen folgende Informationen in die Analysemodelle zur Partnerbeteiligung und zur Dauer des Elterngeldbezugs ein: das Bundesland, das Jahr der Geburt des Kindes und die Anzahl der Kinder im Haushalt. Da die Modelle zur Partnerbeteiligung aufgrund der Datenstruktur ausschließlich Merkmale der Frauen beinhalten, werden zusätzlich der Familienstand in differenzierter Form und die Altersgruppe der Frau berücksichtigt. In den Analysen zur Dauer des väterlichen Elterngeldbezugs wird hingegen der Partnerschaftsstatus im Hinblick auf Ehen und nichteheliche Lebensgemeinschaften unterschieden. Vergleichbar zur Altersgruppe der Frau werden hier die Altersgruppe des Mannes und die Altersdifferenz der Partner einbezogen. Dabei wird angestrebt, gängige und weniger übliche Alterskonstellationen in der Partnerschaft voneinander zu differenzieren.

Zentrale erklärende Variablen sind der Erwerbsstatus und das Erwerbseinkommen beider Partner im Jahr vor der Geburt des Kindes, also zu dem Zeitpunkt, der der Berechnung des Elterngeldes zugrunde gelegt wird. In den Analysen zur Partnerbeteiligung können beide Dimensionen wiederum nur für die Frauen berücksichtigt werden. Bezüglich des Erwerbsstatus erlauben die amtlichen Daten eine Unterscheidung in eine abhängige bzw. selbstständige Erwerbstätigkeit sowie eine Abgrenzung der Nichterwerbszustände Ausbildung, Arbeitslosigkeit und Sonstiges. Die monatlichen Nettoerwerbseinkommen wurden gruppiert, um Unterschiede deutlich hervortreten zu lassen (bis zu 1.000, 1.0012.000, über 2.000 Euro). Da die Analysen zur Dauer des väterlichen Elterngeldbezugs auf vor der Geburt erwerbstätige Väter bzw. auf Doppelverdienerpaare fokussiert sind, wird bezüglich des Erwerbsstatus des Mannes (und der Frau) nur hinsichtlich abhängiger und selbstständiger Erwerbstätigkeit unterschieden. Die Nettoerwerbseinkommen beider Partner finden in den bereits dargestellten Einkommensklassen Eingang in die Analysen. Darüber hinaus wird der genaue Anteil des Erwerbseinkommens der Frau am Erwerbseinkommen des Partners berücksichtigt, um die Verhandlungsposition beider Partner zu bestimmen. Zusätzlich wird die Höhe des gemeinsamen Einkommens beider Partner einbezogen, um mögliche Unterschiede in der Dauer des väterlichen Elterngeldbezugs entlang der Einkommensverteilung deutlich machen zu können. In dem ausschließlich auf Paare in Bayern bezogenen Modell konnte als weiteres Erhebungsmerkmal der Wohnort be-

7 Eine Beschreibung der verwendeten Samples findet sich in den Tabellen A1 und A2. Aufgrund der unterschiedlichen Bevölkerungsdichte und Altersstruktur in den Bundesländern konzentrieren sich drei Viertel aller Partneranträge auf Bayern. 
rücksichtigt werden: Unterschieden werden München, weitere Großstädte und Ortschaften unterhalb der Großstadtgrenze.

Für die Auswertungen des ALLBUS wurden aus den kumulierten Daten der Jahre 1980 bis $2010^{8}$ Informationen zu Einstellungen aus den Jahren 1991, 1992, 2000, 2004 und 2008 für in den Bundesländern Bayern, Mecklenburg-Vorpommern und SchleswigHolstein lebende Männer genutzt. Egalitäre Vorstellungen zur Rollenverteilung zwischen den Geschlechtern ergeben sich aus der Ablehnung von drei klassischen mittels einer vierstufigen Skala erhobenen Items: „Es ist für alle Beteiligten viel besser, wenn der Mann voll im Berufsleben steht und die Frau zu Hause bleibt und sich um den Haushalt und die Kinder kümmert“, „Für eine Frau ist es wichtiger, ihrem Mann bei seiner Karriere zu helfen, als selbst Karriere zu machen“, „Eine verheiratete Frau sollte auf eine Berufstätigkeit verzichten, wenn es nur eine begrenzte Anzahl von Arbeitsplätzen gibt, und wenn ihr Mann in der Lage ist, für den Unterhalt der Familie zu sorgen“. Der Anteil egalitärer Äußerungen wurde über die drei Indikatoren hinweg zusammengefasst. Gleiches gilt für die Einstellungen zu den Konsequenzen der Frauenerwerbstätigkeit, die folgendermaßen erhoben wurden: „Ein Kleinkind wird sicherlich darunter leiden, wenn seine Mutter berufstätig ist“" ,Eine berufstätige Mutter kann ein genauso herzliches und vertrauensvolles Verhältnis zu ihren Kindern finden wie eine Mutter, die nicht berufstätig ist“, „Es ist für ein Kind sogar gut, wenn seine Mutter berufstätig ist und sich nicht nur auf den Haushalt konzentriert". Eine Ablehnung des ersten Indikators und eine Zustimmung zu den letzten beiden Items wurden als eine positive Sicht auf die Auswirkungen der Müttererwerbstätigkeit gewertet.

\subsection{Methode}

Für die empirischen Analysen der jeweils dichotomen abhängigen Variablen werden binäre logistische Regressionen genutzt. Bei der Ermittlung der Determinanten eines Partnerantrags werden zunächst die soziodemographischen Informationen der Frau in das Modell aufgenommen. Dann werden sukzessive die ökonomischen Charakteristika eingeführt, um deren Einfluss auf die Wahrscheinlichkeit eines Partnerantrags zu veranschaulichen. Die Regressionsmodelle zur Analyse der Bezugsdauer des Elterngeldes beziehen sich auf Paare mit einem vor der Geburt des Kindes erwerbstätigen Mann. Zunächst wird der Einfluss der Erwerbsform des Mannes (selbstständig bzw. abhängig beschäftigt) und des Erwerbsstatus der Frau auf die Dauer des Elterngeldbezugs des Mannes überprüft. Dann steht der Einfluss der Erwerbseinkommen beider Partner auf die Bezugsdauer des Elterngeldes durch den Mann im Zentrum des Interesses. Diese Analysen beschränken sich auf Paare, bei denen beide Partner vor der Geburt des Kindes erwerbstätig waren, da hier verhandlungstheoretische Annahmen am ehesten zutreffen sollten. Des Weiteren wird durch die Berücksichtigung von Interaktionstermen mit dem Bundesland überprüft,

8 GESIS - Leibniz-Institut für Sozialwissenschaften (2012): Allgemeine Bevölkerungsumfrage der Sozialwissenschaften ALLBUS 1980-2010. GESIS Datenarchiv, Köln. ZA4574 Version 1.0.1 doi:10.4232/1.11486. 
ob die Entscheidung für einen Partnerantrag bzw. über die Dauer des Elterngeldbezugs mit den regionalen Opportunitätsstrukturen variiert. ${ }^{9}$

Angesichts der zunehmenden Kritik an der Darstellung der Ergebnisse logistischer Regressionen durch odds ratios (Auspurg/Hinz 2011; Best/Wolf 2010, 2012; Mood 2010) wird hier darauf verzichtet. Anstelle dessen werden die Schätzergebnisse durch unstandardisierte Koeffizienten und durchschnittliche marginale Effekte ${ }^{10}$ repräsentiert. Letztere haben gegenüber odds ratios den Vorteil der Robustheit gegenüber unbeobachteter Heterogenität und einer intuitiven Interpretierbarkeit als durchschnittlicher additiver Effekt einer unabhängigen Variablen auf die Wahrscheinlichkeit des interessierenden Ereignisses (Best/Wolf 2010: 840).

\section{Ergebnisse}

\subsection{Determinanten eines Partnerantrags}

Im bundesweiten Vergleich der Wirtschaftskraft liegt Bayern (BY) ganz vorn, während die beiden anderen für die Analyse des Elterngeldbezugs ausgewählten norddeutschen Flächenländer Mecklenburg-Vorpommern (MV) und Schleswig-Holstein (SH) auf den hinteren Plätzen rangieren (Initiative Neue Soziale Marktwirtschaft 2012). MV und SH gelten als ländlich geprägte und strukturschwache Bundesländer, während BY wirtschaftliche Dynamik verkörpert. Im Untersuchungszeitraum von 2007 bis 2009 war die Arbeitslosenquote in MV mit 14 bis 16 Prozent fast doppelt so hoch wie in SH und etwa dreimal so hoch wie in BY (Bundesagentur für Arbeit 2010: 178). Die unterschiedliche Einkommenssituation in den Bundesländern spiegelt sich auch in der durchschnittlichen Höhe des Elterngeldes und im Anteil derjenigen, die den Minimal- bzw. Maximalbetrag erhielten, wider. Für im Jahr 2009 geborene Kinder betrug der durchschnittliche Anspruch auf Elterngeld bei Vätern in BY 1.236 Euro, in MV 988 Euro und in SH 1.145 Euro (Statistisches Bundesamt 2012b: 31). Insofern sind die hier vorgestellten Ergebnisse auch Ausdruck regional variierender Opportunitätsstrukturen.

Wie im gesamten Bundesgebiet wurde auch in den hier beispielhaft untersuchten Bundesländern für die Mehrheit der in den Jahren 2007 bis 2009 geborenen Kinder kein Partnerantrag gestellt (Abbildung 1).

9 Die Ergebnisse dieser Analysen werden nur im Falle signifikanter Interaktionseffekte ausgewiesen.

10 Dieser Durchschnittseffekt (Average Marginal Effect, AME) ist der Mittelwert der marginalen Effekte über alle Beobachtungen hinweg (Best/Wolf 2010: 840). 
Abbildung 1: $\quad$ Partneranträge eines Elterngeldbezugs für Geburten in den Jahren 2007 bis 2009 und dessen Dauer

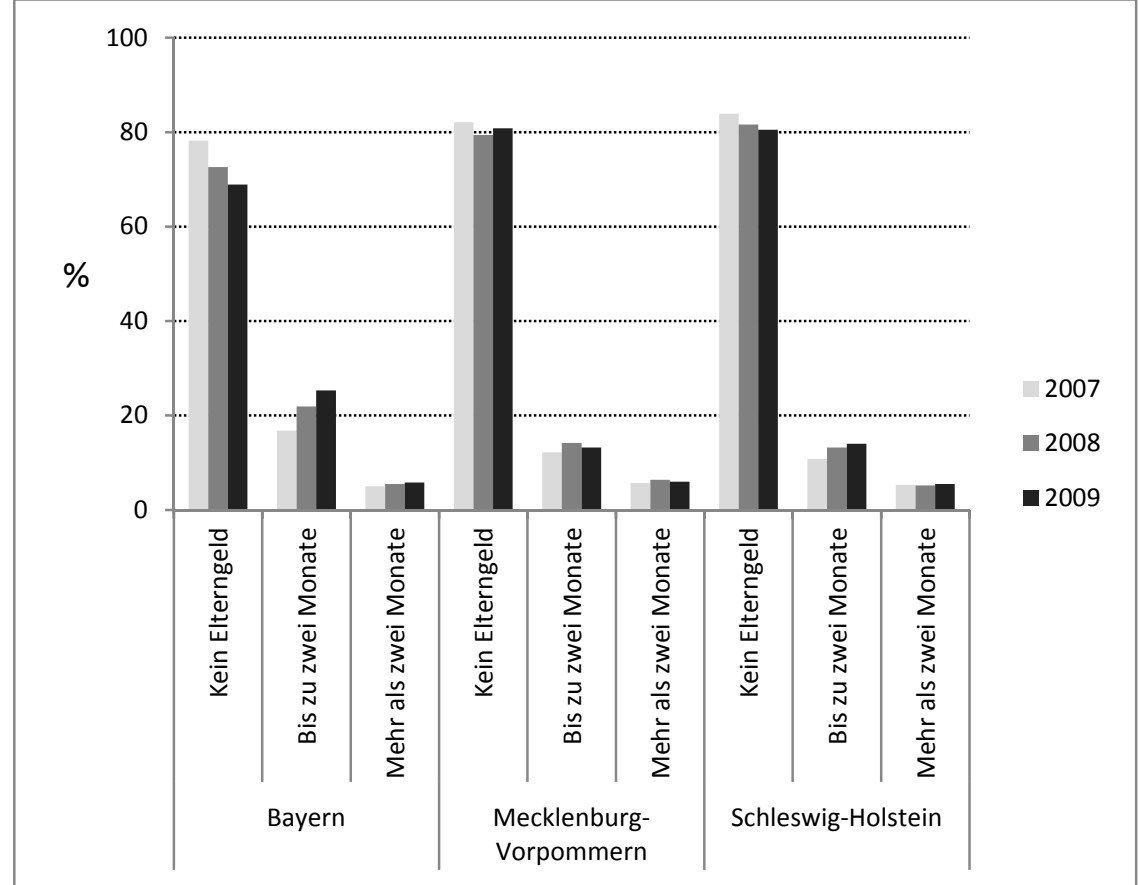

Quelle: Daten des Elterngeldbezugs in BY bereitgestellt durch das Zentrum Bayern Familie und Soziales, Daten des Elterngeldbezugs in MV bereitgestellt durch das Landesamt für Gesundheit und Soziales (LAGuS), Daten des Elterngeldbezugs in SH bereitgestellt durch das Landesamt für soziale Dienste, eigene Berechnungen.

Am ehesten wurde in BY für einen Zeitraum von bis zu zwei Monaten ein Partnerantrag gestellt, während ein längerer Elterngeldbezug eine Seltenheit darstellt und hier auch keine dynamische Entwicklung im Zeitverlauf zu verzeichnen ist. Die sich auch in der Elterngeldstatistik abzeichnende zunehmende Väterbeteiligung konzentriert sich ausschließlich auf die beiden ,Bonusmonate“. 11

11 In dieser Darstellung sind auch Frauen enthalten, die sich vom Partner ihres Kindes getrennt haben oder die nie eine Partnerschaft eingegangen sind. 
Tabelle 1: Determinanten eines Partnerantrags in BY, MV und SH

\begin{tabular}{|c|c|c|c|c|}
\hline & \multicolumn{2}{|c|}{ Modell 1} & \multicolumn{2}{|c|}{ Modell 2} \\
\hline & Koeff. (SE) & AME & Koeff. (SE) & AME \\
\hline \multicolumn{5}{|l|}{ Bundesland } \\
\hline Schleswig-Holstein (SH) & - & - & - & - \\
\hline Bayern (BY) & $0,52^{\star \star *}(0,01)$ & 0,09 & $0,50^{* \star *}(0,01)$ & 0,08 \\
\hline Mecklenburg-Vorpommern (MV) & $0,32^{* \star \star}(0,02)$ & 0,06 & $0,31^{* \star \star}(0,02)$ & 0,05 \\
\hline \multicolumn{5}{|l|}{ Jahr der Geburt des Kindes } \\
\hline 2007 & - & - & - & - \\
\hline 2008 & $0,30^{* * *}(0,01)$ & 0,05 & $0,28^{* \star *}(0,01)$ & 0,05 \\
\hline 2009 & $0,44^{* * *}(0,01)$ & 0,07 & $0,41^{* * *}(0,01)$ & 0,07 \\
\hline \multicolumn{5}{|l|}{ Familienstand der Frau } \\
\hline verheiratet & - & - & - & - \\
\hline eingetragene Lebenspartnerschaft & $-0,32^{\star \star \star}(0,08)$ & $-0,06$ & $-0,33^{\star \star \star}(0,08)$ & $-0,06$ \\
\hline ledig & $-0,33^{* \star *}(0,01)$ & $-0,06$ & $-0,33^{\star \star *}(0,01)$ & $-0,06$ \\
\hline verwitwet/geschieden & $-0,70^{* \star *}(0,03)$ & $-0,12$ & $-0,71^{\star \star *}(0,03)$ & $-0,12$ \\
\hline keine Angabe & $-0,43^{* * t}(0,03)$ & $-0,07$ & $-0,39^{* * t}(0,03)$ & $-0,07$ \\
\hline \multicolumn{5}{|l|}{ Anzahl der Kinder im Haushalt } \\
\hline 1 Kind & - & - & - & - \\
\hline 2 Kinder & $-0,27^{* \star *}(0,01)$ & $-0,05$ & $-0,06^{* \star *}(0,01)$ & $-0,01$ \\
\hline $3+$ Kinder & $-0,47^{\star \star *}(0,01)$ & $-0,08$ & $-0,22^{* * t}(0,01)$ & $-0,04$ \\
\hline \multicolumn{5}{|l|}{ Altersgruppe der Frau } \\
\hline unter 30 Jahre & - & - & - & - \\
\hline 30 bis 34 Jahre & $0,56^{\star \star \star}(0,01)$ & 0,09 & $0,42^{\star \star \star}(0,01)$ & 0,07 \\
\hline 35 Jahre und älter & $0,65^{* * *}(0,01)$ & 0,11 & $0,48^{* * *}(0,01)$ & 0,08 \\
\hline \multicolumn{5}{|c|}{ Erwerbsstatus der Frau ein Jahr vor der Geburt } \\
\hline abhängig erwerbstätig & - & - & & \\
\hline selbstständig & $0,35^{\star \star *}(0,02)$ & 0,06 & & \\
\hline in Ausbildung & $-0,12^{* \star *}(0,03)$ & $-0,02$ & & \\
\hline Hausfrau & $-0,75^{* \star \star}(0,01)$ & $-0,13$ & & \\
\hline arbeitslos & $-1,15^{\star \star \star}(0,02)$ & $-0,20$ & & \\
\hline
\end{tabular}

Erwerbseinkommen der Frau ein Jahr vor der Geburt

kein Erwerbseinkommen

\begin{tabular}{lll} 
bis zu 1.000 Euro & $0,41^{* * *}(0,01)$ & 0,07 \\
$1.001-2.000$ Euro & $1,05^{* * *}(0,01)$ & 0,18 \\
mehr als 2.000 Euro & $1,40^{* * *}(0,02)$ & 0,23 \\
\hline
\end{tabular}

\begin{tabular}{lcc} 
mehr als 2.000 Euro & $1,40(0,02)$ & 0,23 \\
Konstante & $-1,81^{* * *}(0,01)$ & $-2,56^{* * *}(0,02)$ \\
\hline Likelihood Ratio & 26939,20 & 31784,52 \\
Df & 16 & 15 \\
Cox \& Snell R & 0,06 & 0,08 \\
N mit Elterngeldbezug / N & $97.312 / 406.616$ & $97.312 / 406.616$ \\
\hline
\end{tabular}

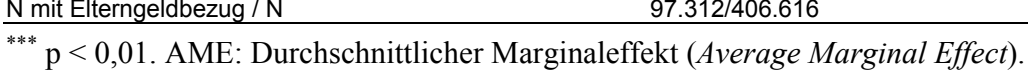

Quelle: Daten des Elterngeldbezugs in BY bereitgestellt durch das Zentrum Bayern Familie und Soziales, Daten des Elterngeldbezugs in MV bereitgestellt durch das Landesamt für Gesundheit und Soziales (LAGuS), Daten des Elterngeldbezugs in SH bereitgestellt durch das Landesamt für soziale Dienste, eigene Berechnungen.

Auch in den multivariaten Analysen haben die deskriptiven Befunde Bestand (Tabelle 1). In BY und MV ist es wahrscheinlicher einen Partnerantrag zu stellen als in SH. Vom Ge- 
burtsjahr des Kindes geht ein positiver Effekt auf den Elterngeldbezug durch einen Partner aus. Interessant ist, dass jeglicher Familienstand, der von der Ehe abweicht, einen negativen Effekt auf die Wahrscheinlichkeit eines Partnerantrags hat.

Dies gilt auch für die eingetragene Lebenspartnerschaft zweier Frauen und in besonders starkem Maße für verwitwete und geschiedene Frauen. ${ }^{12}$ Mit der Anzahl der Kinder im Haushalt sinkt die Wahrscheinlichkeit eines Partnerantrags. Mit steigendem Alter der Frau wird ein Partnerantrag hingegen wahrscheinlicher, was möglicherweise mit einer stabileren Positionierung der Frau und ihres Partners im Erwerbssystem zusammenhängt. Von einer selbstständigen Erwerbstätigkeit der Frau geht ein positiver Effekt auf einen Partnerantrag aus, während alle Nichterwerbsphasen und hier insbesondere eine Arbeitslosigkeit einen negativen Effekt haben. Verglichen mit einer abhängigen Beschäftigung senkt eine Arbeitslosigkeit der Frau die Wahrscheinlichkeit eines Partnerantrags um durchschnittlich 20 Prozentpunkte (Modell 1). Mit zunehmender Höhe des Erwerbseinkommens der Frau erhöht sich die Wahrscheinlichkeit eines Elterngeldbezugs des Partners. Von den ökonomischen Ressourcen der Frau geht demnach ein positiver Effekt auf die Wahrscheinlichkeit einer Partnerbeteiligung am Elterngeld aus.

\subsection{Determinanten eines längeren Elterngeldbezugs durch den Partner}

In Übereinstimmung mit haushaltsökonomischen Erwägungen beziehen Väter am ehesten länger als zwei Monate Elterngeld, wenn sie vor der Geburt des Kindes nicht erwerbstätig waren (30 Prozent der nicht erwerbstätigen Männer verglichen mit 19 Prozent der Väter mit Partnerantrag insgesamt). Jedoch umfasst die Gruppe der vor der Geburt nicht erwerbstätigen Väter nur fünf Prozent der Männer mit Elterngeldbezug, so dass im Folgenden eine Konzentration auf die vor der Geburt erwerbstätigen Väter erfolgt. Nur für diese Gruppe ist ein Elterngeldbezug auch mit einer Einschränkung der Erwerbstätigkeit im Sinne einer Elternzeit verbunden.

Abbildung 2 verdeutlicht wie Paare, die einen Partnerantrag gestellt haben, die Zeit des Elterngeldbezugs aufteilen. Prinzipiell ist eine beliebige Aufteilung der Zeit des Elterngeldbezugs innerhalb der Partnerschaft möglich. Die Elternzeit kann auch gemeinsam genommen werden, was nach Angaben der Elterngeldstatistik von etwas zwei Dritteln der Paare zeitweise praktiziert wird (Statistisches Bundesamt 2012a). Während der Zeit des Elterngeldbezugs ist eine Teilzeitarbeit von bis zu 30 Wochenstunden möglich (BMFSFJ 2012c). Die Maximaldauer des Elterngeldbezugs von 14 Monaten kann jedoch nur ausgeschöpft werden, wenn ein Partner mindestens zwei Monate Elterngeld bezieht.

12 Diese Frauen haben möglicherweise ebenso wie ledige Frauen keinen aktuellen Partner. 
Abbildung 2: Aufteilung der Bezugsmonate des Elterngeldes innerhalb der Partnerschaft (Paare mit erwerbstätigem Mann, Geburten 2007-2009)

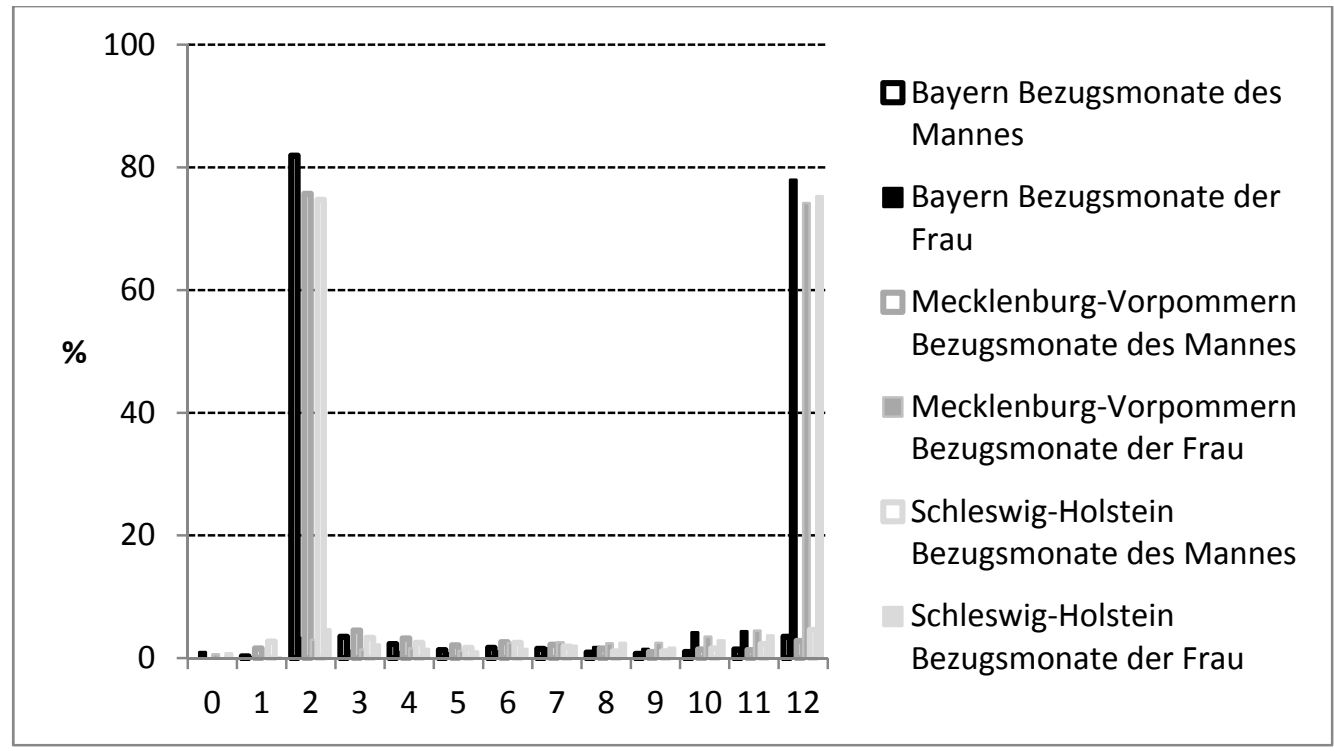

Quelle: Daten des Elterngeldbezugs in BY bereitgestellt durch das Zentrum Bayern Familie und Soziales, Daten des Elterngeldbezugs in MV bereitgestellt durch das Landesamt für Gesundheit und Soziales (LAGuS), Daten des Elterngeldbezugs in SH bereitgestellt durch das Landesamt für soziale Dienste, eigene Berechnungen.

Wie Abbildung 2 sehr deutlich zeigt, entscheiden sich mehr als drei Viertel aller Paare für eine Kombination von genau 12 Monaten, die durch die Frau und zwei Monaten, die durch den Mann genommen werden. Besonders ausgeprägt, ist dieses typische, nahezu normative Muster in BY. Alle anderen Kombinationen, bei denen die Frau einen Teil ,ihrer" Elternzeit an den Partner abgeben müsste, sind eher selten. Interessant ist, dass in einer im Jahr 2012 durchgeführten repräsentativen Väterstudie ein Drittel der Befragten angab, dass sie gar nicht wussten, dass sie mehr als zwei Monate Elternzeit hätten nehmen können bzw. dass sie sich darüber unsicher waren (Väter gGmbH 2012: 48). Vor diesem Hintergrund wird nun gefragt, in welcher Weise ökonomische Ressourcen des Paares einen über die Zweimonatsnorm hinausgehenden Elterngeldbezug des Partners beeinflussen.

Das multivariate Modell zur Überprüfung des Einflusses des Erwerbsstatus (Tabelle 2, Modell 1) weist aus, dass ein längerer Elterngeldbezug des Partners in BY signifikant unwahrscheinlicher ist als in SH, während sich dieser in MV nur unwesentlich von SH unterscheidet. 
Tabelle 2: Determinanten eines mehr als zweimonatigen Elterngeldbezugs durch vor der Geburt des Kindes erwerbstätige Partner in BY, MV und SH

\begin{tabular}{|c|c|c|c|c|}
\hline & \multicolumn{2}{|c|}{$\begin{array}{l}\text { Alle Paare } \\
\text { Modell } 1\end{array}$} & \multicolumn{2}{|c|}{$\begin{array}{c}\text { Nur Paare in Bayern } \\
\text { Modell } 2\end{array}$} \\
\hline & Koeff. (SE) & AME & Koeff. (SE) & AME \\
\hline \multicolumn{5}{|l|}{ Bundesland } \\
\hline Schleswig-Holstein (SH) & - & - & & \\
\hline Bayern (BY) & $-0,32^{* * \star}(0,03)$ & $-0,05$ & & \\
\hline Mecklenburg-Vorpommern (MV) & $-0,04(0,04)$ & $-0,01$ & & \\
\hline \multicolumn{5}{|l|}{ Wohnort } \\
\hline keine Großstadt & & & - & - \\
\hline München & & & $0,48^{* \star \star}(0,03)$ & 0,07 \\
\hline andere Großstadt & & & $0,30^{* * \star}(0,03)$ & 0,04 \\
\hline \multicolumn{5}{|l|}{ Jahr der Geburt des Kindes } \\
\hline 2007 & - & - & - & - \\
\hline 2008 & $-0,14^{\star \star \star}(0,02)$ & $-0,02$ & $-0,16^{\star \star \star}(0,02)$ & $-0,02$ \\
\hline$\underline{2009}$ & $-0,20^{\star * \star}(0,02)$ & $-0,03$ & $-0,24^{* \star \star}(0,02)$ & $-0,03$ \\
\hline \multicolumn{5}{|l|}{ Partnerschaftsform } \\
\hline verheiratet & - & - & - & - \\
\hline nichteheliche Lebensgemeinschaft & $0,19^{* * *}(0,02)$ & 0,03 & $0,21^{* * * *}(0,03)$ & 0,03 \\
\hline \multicolumn{5}{|l|}{ Anzahl der Kinder im Haushalt } \\
\hline 1 Kind & - & - & - & - \\
\hline 2 Kinder & $0,25^{\star \star \star}(0,02)$ & 0,04 & $0,31^{* \star \star}(0,02)$ & 0,04 \\
\hline $3+$ Kinder & $0,43^{* * *}(0,03)$ & 0,06 & $0,50^{* \star \star}(0,03)$ & 0,07 \\
\hline \multicolumn{5}{|l|}{ Altersgruppe des Mannes } \\
\hline unter 30 Jahre & - & - & - & - \\
\hline 30 bis 34 Jahre & $-0,06(0,03)$ & $-0,01$ & $-0,10^{\star \star \star}(0,04)$ & $-0,01$ \\
\hline 35 Jahre und älter & $0,19^{* * *}(0,03)$ & 0,03 & $0,14^{\star \star \star}(0,04)$ & 0,02 \\
\hline \multicolumn{5}{|l|}{ Altersdifferenz der Partner } \\
\hline Mann gleichaltrig o. bis $3 \mathrm{~J}$. älter als Frau & - & - & - & - \\
\hline Mann mehr als 3 Jahre älter als Frau & $0,01(0,02)$ & 0,001 & $0,02(0,02)$ & 0,002 \\
\hline Mann mindestens ein Jahr jünger als Frau & $0,10^{* * \star}(0,03)$ & 0,01 & $0,07(0,03)$ & 0,01 \\
\hline \multicolumn{5}{|c|}{ Erwerbsstatus der Frau ein Jahr vor der Geburt } \\
\hline abhängig erwerbstätig & - & - & - & - \\
\hline selbstständig & $1,05^{* * *}(0,04)$ & 0,15 & $0,99^{* * *}(0,04)$ & 0,14 \\
\hline in Ausbildung & $1,69^{\star \star \star}(0,07)$ & 0,25 & $1,75^{\star \star \star}(0,10)$ & 0,25 \\
\hline Hausfrau & $0,69^{\star \star \star}(0,02)$ & 0,10 & $0,68^{\star \star *}(0,03)$ & 0,10 \\
\hline arbeitslos & $0,41^{* * *}(0,07)$ & 0,06 & $0,51^{* \star *}(0,09)$ & 0,07 \\
\hline
\end{tabular}

Erwerbsstatus des Mannes ein Jahr vor der Geburt

abhängig erwerbstätig

\begin{tabular}{|c|c|c|c|c|}
\hline selbstständig & $0,06(0,03)$ & 0,01 & $0,11^{* * *}(0,03)$ & 0,02 \\
\hline Konstante & $-1,59^{* * *}(0,04$ & & \multicolumn{2}{|l|}{$-1,99^{* * \star}(0,04)$} \\
\hline Likelihood Ratio & \multicolumn{2}{|c|}{3186,29} & \multicolumn{2}{|c|}{2823,67} \\
\hline Df & \multicolumn{2}{|c|}{16} & \multicolumn{2}{|c|}{16} \\
\hline Cox \& Snell $\mathrm{R}^{2}$ & \multicolumn{2}{|c|}{0,03} & \multicolumn{2}{|c|}{0,04} \\
\hline $\mathrm{N}$ mit Elterngeldbezug / $\mathrm{N}$ & 16.8 & 0.755 & \multicolumn{2}{|c|}{$13.352 / 75.279$} \\
\hline
\end{tabular}

$\mathrm{N}$ mit Elterngeldbezug / $\mathrm{N}$

ekt (Average Marginal Effect).

Quelle: Daten des Elterngeldbezugs in BY bereitgestellt durch das Zentrum Bayern Familie und Soziales, Daten des Elterngeldbezugs in MV bereitgestellt durch das Landesamt für Gesundheit und Soziales 
(LAGuS), Daten des Elterngeldbezugs in SH bereitgestellt durch das Landesamt für soziale Dienste, eigene Berechnungen.

Unter Gleichstellungsgesichtspunkten ist besonders bedenkenswert, dass es eine negative Dynamik im Zeitverlauf gibt, d.h. für im Jahr 2009 geborene Kinder war eine längere Elternzeit des Vaters weniger wahrscheinlich als für im Jahr 2007 geborene Kinder. Ein nichteheliches Zusammenleben der Eltern erhöht die Wahrscheinlichkeit eines längeren Elterngeldbezugs des Vaters, was ein Hinweis auf eine geringere geschlechtsspezifische Spezialisierung in nichtehelichen Lebensgemeinschaften ist (Beblo 2012). Gleiches gilt für ein Kind höherer Ordnungszahl. Letzteres könnte auf positive Erfahrungen mit dem ersten Kind hindeuten, darauf, dass Frauen ihren Partnern bei weiteren Kindern eher zutrauen, diese adäquat zu betreuen oder auf eine erforderliche Entlastung der Partnerin. Väter ab 35 Jahren haben eine signifikant höhere Wahrscheinlichkeit einer längeren Elternzeit als jüngere Väter, was wiederum für eine stabile Erwerbsintegration sprechen könnte. Auch ein im Vergleich zur Partnerin jüngeres Alter des Mannes begünstigt einen längeren Elterngeldbezug. Interessant ist, dass, verglichen mit einer abhängigen Erwerbstätigkeit der Frau im Jahr vor der Geburt des Kindes, alle anderen Erwerbszustände eine längere Elternzeit des Partners positiv beeinflussen. In besonderem Maße gilt dies, wenn die Frau in einer Ausbildung oder selbstständig beschäftigt ist, was eine spezielle Unterstützung durch den Partner in diesen Lebenssituationen signalisiert. Vor dem Hintergrund ökonomischer Überlegungen erstaunt jedoch, dass eine abhängige Erwerbstätigkeit der Frau lediglich einen kürzeren Elterngeldbezug des Partners befördert. Gerade bei Doppelverdienerpaaren ist die „12+2-Norm“ besonders stark ausgeprägt. Dies deutet darauf hin, dass Frauen diese einjährige Unterbrechung ihrer Erwerbstätigkeit vielleicht auch als legitim betrachten und nicht bereit sind, diese zugunsten ihres Partners zu reduzieren. In diese Richtung weisen auch die Ergebnisse der Väterstudie, in der zwei Drittel der Väter, die Elternzeit genommen hatten, angaben, dass ihre Frau in jedem Fall 12 Monate Elternzeit nehmen wollte (Väter gGmbH 2012: 47). Der nicht signifikante Einfluss einer selbstständigen Erwerbstätigkeit des Mannes auf die Wahrscheinlichkeit eines längeren Elterngeldbezugs verdeckt einen interessanten Interaktionseffekt mit dem Bundesland. In zusätzlichen Analysen wurde deutlich, dass in BY von einer selbstständigen Tätigkeit ein positiver Effekt ausgeht, während dieser in den beiden anderen Bundesländern negativ ist. Dies hängt sicherlich mit dem regional variierenden Charakter der Selbstständigkeit zusammen, der unterschiedliche Handlungsspielräume eröffnet (z.B. Soloselbstständigkeit verglichen mit der Führung eines kleinen Unternehmens).

Modell 2 (Tabelle 2) beschränkt sich auf in BY lebende Paare und bestätigt viele der bereits dargestellten Effekte. Zusätzlich kann hier der Wohnort berücksichtigt werden. Großstädtisches Leben, insbesondere in München, wirkt sich positiv auf einen längeren Elterngeldbezug von Vätern aus. Dies kann sowohl ein Effekt entsprechender Einstellungen der Eltern als auch entsprechender Gelegenheiten, beispielsweise eines höheren Anteils von Vätern im öffentlichen Dienst, sein. ${ }^{13}$ Darüber hinaus zeigt sich ein etwas anderer Effekt des Alters des Mannes: Ein mittleres Alter wirkt sich, verglichen mit einem jüngeren Alter, signifikant negativ auf einen längeren Elterngeldbezug aus.

13 In zusätzlichen Analysen wurde ermittelt, dass es sich bei dem „Großstadteffekt“ nicht um einen Effekt des Einkommens handelt, denn er bleibt auch nach Kontrolle des Einkommens signifikant. 
Tabelle 3: Determinanten eines mehr als zweimonatigen Elterngeldbezugs bei Doppelverdienerpaaren in BY, MV und SH

\begin{tabular}{|c|c|c|c|c|c|c|}
\hline & \multicolumn{2}{|c|}{ Modell 1} & \multicolumn{2}{|c|}{ Modell 2} & \multicolumn{2}{|c|}{ Modell 3} \\
\hline & Koeff. (SE) & AME & Koeff. (SE) & AME & Koeff. (SE) & AME \\
\hline \multicolumn{7}{|l|}{ Bundesland } \\
\hline Schleswig-Holstein (SH) & - & - & - & - & - & - \\
\hline Bayern (BY) & $-0,38^{* * *}(0,03)$ & $-0,05$ & $-0,37^{* \star *}(0,03)$ & $-0,05$ & $-0,36^{\star \star \star}(0,03)$ & $-0,05$ \\
\hline Mecklenburg-Vorpommern (MV) & $0,03(0,05)$ & 0,004 & $0,05(0,05)$ & 0,01 & $-0,01(0,05)$ & $-0,00$ \\
\hline \multicolumn{7}{|l|}{ Jahr der Geburt des Kindes } \\
\hline 2007 & - & - & - & - & - & - \\
\hline 2008 & $-0,12^{\star \star \star}(0,03)$ & $-0,02$ & $-0,12^{\star * *}(0,03)$ & $-0,02$ & $-0,12^{\star \star \star}(0,03)$ & $-0,02$ \\
\hline$\underline{2009}$ & $-0,20^{* * *}(0,02)$ & $-0,03$ & $-0,20^{* * *}(0,02)$ & $-0,03$ & $-0,21^{* * *}(0,02)$ & $-0,03$ \\
\hline \multicolumn{7}{|l|}{ Partnerschaftsform } \\
\hline verheiratet & - & - & - & - & - & - \\
\hline nichteheliche Lebensgemeinschaft & $0,21^{* * \star}(0,02)$ & 0,03 & $0,23^{* * \star}(0,02)$ & 0,03 & $0,15^{\star \star \star}(0,02)$ & 0,02 \\
\hline \multicolumn{7}{|l|}{ Anzahl der Kinder im Haushalt } \\
\hline 1 Kind & - & - & - & - & - & - \\
\hline 2 Kinder & $0,19^{\star \star \star}(0,02)$ & 0,02 & $0,13^{* * *}(0,02)$ & 0,02 & $0,32^{* \star *}(0,02)$ & 0,04 \\
\hline $3+$ Kinder & $0,42^{* * \star}(0,04)$ & 0,06 & $0,34^{* * *}(0,04)$ & 0,04 & $0,55^{\star * \star}(0,03)$ & 0,07 \\
\hline \multicolumn{7}{|l|}{ Altersgruppe des Mannes } \\
\hline unter 30 Jahre & - & - & - & - & - & - \\
\hline 30 bis 34 Jahre & $-0,02(0,04)$ & $-0,00$ & $-0,04(0,04)$ & $-0,01$ & $-0,03(0,04)$ & $-0,00$ \\
\hline 35 Jahre und älter & $0,19^{* * *}(0,04)$ & 0,03 & $0,20^{* \star *}(0,04)$ & 0,03 & $0,20^{* * \star}(0,04)$ & 0,03 \\
\hline \multicolumn{7}{|l|}{ Altersdifferenz der Partner } \\
\hline M. gleichaltrig o. b. 3 J. älter als F. & - & - & - & - & - & - \\
\hline M. mehr als 3 Jahre älter als F. & $0,02(0,02)$ & 0,002 & $0,002(0,02)$ & 0,00 & $0,01(0,02)$ & 0,00 \\
\hline M. mind. ein Jahr jünger als F. & $0,10^{* * *}(0,03)$ & 0,01 & $0,10^{* \star *}(0,03)$ & 0,01 & $0,10^{* * \star}(0,03)$ & 0,01 \\
\hline \multicolumn{7}{|c|}{ Erwerbsstatus der Frau ein Jahr vor der Geburt } \\
\hline abhängig erwerbstätig & - & - & - & - & - & - \\
\hline selbstständig & $0,95^{\star * \star}(0,04)$ & 0,13 & $1,03^{* \star *}(0,04)$ & 0,14 & $1,04^{\star \star *}(0,04)$ & 0,14 \\
\hline \multicolumn{7}{|c|}{ Erwerbsstatus des Mannes Frau ein Jahr vor der Geburt } \\
\hline abhängig erwerbstätig & - & - & - & - & - & - \\
\hline selbstständig & $-0,06(0,03)$ & $-0,01$ & $-0,06(0,03)$ & $-0,01$ & $-0,06(0,03)$ & $-0,01$ \\
\hline \multicolumn{7}{|c|}{ Erwerbseinkommen der Frau ein Jahr vor der Geburt } \\
\hline bis $z u € 1.000$ & $0,66^{* * *}(0,02)$ & 0,09 & & & & \\
\hline$€ 1.001-2.000$ & - & - & & & & \\
\hline$€ 2.000+$ & $0,62^{* * *}(0,03)$ & 0,08 & & & & \\
\hline \multicolumn{7}{|c|}{ Erwerbseinkommen des Mannes ein Jahr vor der Geburt } \\
\hline bis $z u € 1.000$ & $-0,09^{* * *}(0,03)$ & $-0,01$ & $0,03(0,04)$ & 0,00 & $-0,30^{\star \star \star}(0,04)$ & $-0,04$ \\
\hline$€ 1.001-2.000$ & - & - & - & - & - & - \\
\hline$€ 2.000+$ & $0,15^{\star * \star}(0,02)$ & 0,02 & $0,11^{* * *}(0,02)$ & 0,01 & $0,25^{* * *}(0,03)$ & 0,03 \\
\hline \multicolumn{7}{|c|}{ Anteil des Erwerbseinkommens der Frau am Erwerbseinkommen des Partners } \\
\hline$<50 \%$ & & & - & - & & \\
\hline 50 bis $79 \%$ & & & $-0,73^{\star \star \star}(0,03)$ & $-0,10$ & & \\
\hline 80 bis $119 \%$ & & & $-0,58^{* * *}(0,03)$ & $-0,08$ & & \\
\hline$\geq 120 \%$ & & & $-0,46^{* * *}(0,04)$ & $-0,06$ & & \\
\hline
\end{tabular}




\begin{tabular}{|c|c|c|c|c|c|c|}
\hline & \multicolumn{2}{|c|}{ Modell 1} & \multicolumn{2}{|c|}{ Modell 2} & \multicolumn{2}{|c|}{ Modell 3} \\
\hline & Koeff. (SE) & AME & Koeff. (SE) & AME & Koeff. (SE) & AME \\
\hline \multicolumn{7}{|c|}{ Summe der Erwerbseinkommen beider Partner } \\
\hline bis $z u € 2.000$ & & & & & - & - \\
\hline$€ 2.001-3.000$ & & & & & $-0,56^{\star \star \star}(0,04)$ & $-0,07$ \\
\hline$€ 3.001-4.000$ & & & & & $-0,57^{\star * *}(0,04)$ & $-0,08$ \\
\hline$€ 4.000+$ & & & & & $-0,51^{\star * *}(0,05)$ & $-0,07$ \\
\hline Konstante & $-1,97^{* * *}(0,05)$ & & $-1,18^{* * *}(0,05)$ & & $-1,18^{*+* t}(0,06)$ & \\
\hline Likelihood Ratio & \multicolumn{2}{|c|}{2907,40} & \multicolumn{2}{|l|}{2687,14} & \multicolumn{2}{|c|}{2202,85} \\
\hline Df & \multicolumn{2}{|c|}{17} & \multicolumn{2}{|c|}{18} & \multicolumn{2}{|c|}{18} \\
\hline Cox \& Snell $\mathrm{R}^{2}$ & \multicolumn{2}{|c|}{0,04} & \multicolumn{2}{|c|}{0,03} & \multicolumn{2}{|l|}{0,03} \\
\hline $\mathrm{N}$ mit Elterngeldbezug / $\mathrm{N}$ & \multicolumn{2}{|c|}{$12.573 / 76.220$} & \multicolumn{2}{|c|}{$12.573 / 76.220$} & \multicolumn{2}{|c|}{$12.573 / 76.220$} \\
\hline
\end{tabular}

${ }^{* * *} \mathrm{p}<0,01$. AME: Durchschnittlicher Marginaleffekt (Average Marginal Effect).

Quelle: Daten des Elterngeldbezugs in BY bereitgestellt durch das Zentrum Bayern Familie und Soziales, Daten des Elterngeldbezugs in MV bereitgestellt durch das Landesamt für Gesundheit und Soziales (LAGuS), Daten des Elterngeldbezugs in SH bereitgestellt durch das Landesamt für soziale Dienste, eigene Berechnungen.

Tabelle 3 weist Modelle zum Einfluss des vor der Geburt des Kindes erzielten Erwerbseinkommens hinsichtlich eines mindestens vierteljährlichen Elterngeldbezugs des Partners bei Doppelverdienerpaaren aus. Viele der bereits beschriebenen Einflussfaktoren behalten auch bei dieser Begrenzung des Samples ihre Relevanz. Eine selbstständige Erwerbstätigkeit der Frau wirkt sich wiederum positiv auf einen längeren Elterngeldbezug des Partners aus. Verglichen mit einem mittleren Erwerbseinkommen begünstigen sowohl ein hohes Einkommen der Frau als auch ein hohes Einkommen des Mannes einen längeren Elterngeldbezug (Modell 1). Ökonomische Ressourcen beider Partner sind also wichtig, um die Einkommensreduzierung verkraften zu können. Ebenso schlüssig ist, dass ein geringes Einkommen des Mannes einen negativen Effekt hat. Nicht im Einklang mit ökonomischen Überlegungen steht, dass ein geringes Einkommen der Frau ebenfalls positiv auf einen längeren Elterngeldbezug des Partners wirkt. Insgesamt leisten der Erwerbsstatus und das Erwerbseinkommen der Frau einen größeren Erklärungsbeitrag als die ökonomischen Charakteristika des Mannes. Um die Verhandlungsposition der Partner direkt zu operationalisieren, wird in Modell 2 die Einkommensrelation beider Partner berücksichtigt. Entsprechend ressourcentheoretischer Überlegungen sollte eine im Vergleich zur Partnerin günstigere ökonomische Position eine längere Elternzeit des Vaters weniger wahrscheinlich machen. Jedoch zeigt sich empirisch das Gegenteil. Von einem hohen Erwerbseinkommen des Partners geht weiterhin ein positiver Effekt aus und ein höherer Anteil des Einkommens der Frau am Einkommen des Partners erschwert eine längere Elternzeit des Mannes. Einzig wenn ihr Einkommen weniger als die Hälfte seines Einkommens beträgt, ist die Wahrscheinlichkeit eines längeren Elterngeldbezugs des Mannes erhöht. Bei Frauen, die ein vergleichbares oder höheres Einkommen als ihr Partner erzielen, ist das hingegen nicht der Fall, was auch gegen die Annahme spricht, dass gerade in diesen Konstellationen Vorstellungen von engagierter Vaterschaft umgesetzt werden. Frauen in vergleichsweise günstiger ökonomischer Situation scheinen selbst eine maximale Elternzeit anzustreben. In Modell 3 wird anstelle der Einkommensrelation die Summe beider Erwerbseinkommen einbezogen, um Unterschiede entlang der Einkommensverteilung 
zu verdeutlichen. Erneut zeigt sich, dass Männer mit einem höheren Einkommen verstärkt zu einer längeren Elternzeit tendieren. Jedoch geht vor allem von einem geringen gemeinsamen Einkommen beider Partner ein positiver Effekt aus, während sich zwischen den höheren Einkommensklassen kaum Unterschiede zeigen. Es spricht also wenig dafür, dass diejenigen, die einen Einkommensausfall am ehesten ökonomisch verkraften könnten, häufiger eine längere Elternzeit des Vaters realisieren.

\subsection{Einstellungen zu Geschlechterrollen und den Konsequenzen der Frauenerwerbstätigkeit}

Leider kann mit den von den Elterngeldstellen erhobenen Informationen nicht überprüft werden, welche Relevanz Einstellungen für einen längeren Elterngeldbezug von Vätern haben. Da sich gezeigt hat, dass die Dauer der Elternzeit von Vätern regional variiert (vgl. Tabelle A2), sollen im Folgenden auf der Ebene von Bundesländern einige Einstellungsindikatoren auf Basis von ALLBUS-Daten hinzugezogen werden. ${ }^{14}$

Abbildung 3: Anteil moderner Äußerungen von Männern

(kumulierte Wellen 1991-2008)

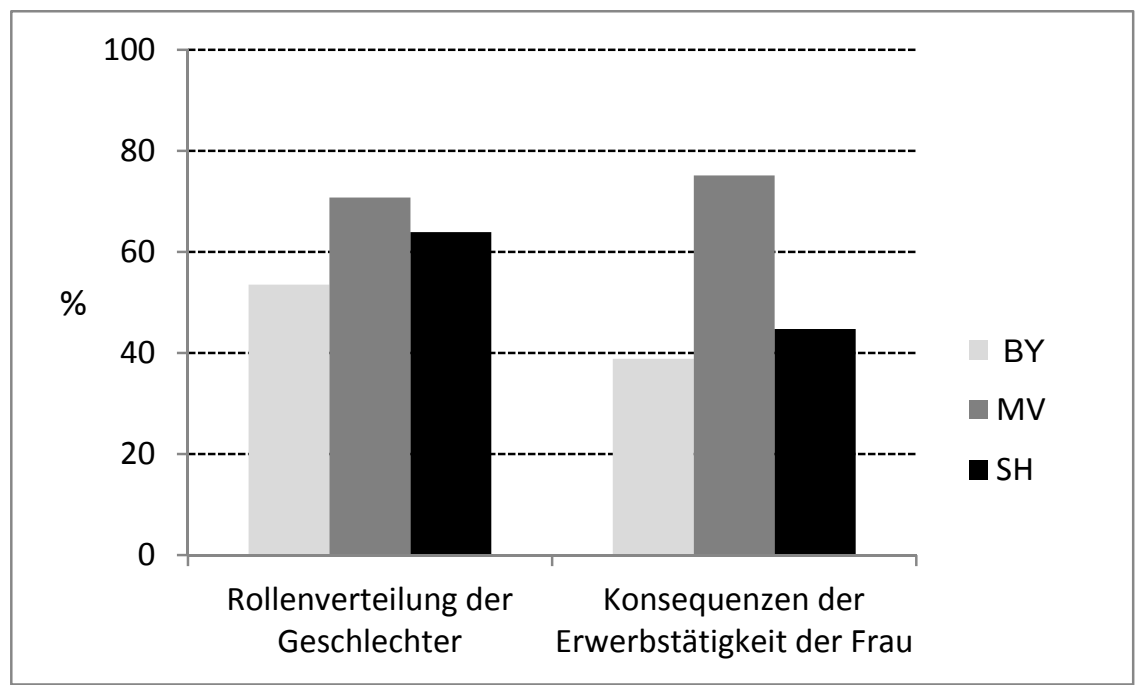

Quelle: ALLBUS 1980-2010.

Abbildung 3 macht deutlich, dass die regional variierenden Unterschiede in den Einstellungen von Männern zur Rollenverteilung der Geschlechter weniger gravierend ausfallen als bezüglich der Einschätzung der Konsequenzen der Müttererwerbstätigkeit. Bei Män-

14 Im Aggregat nachgewiesene Einstellungsunterschiede zwischen in verschiedenen Bundesländern lebenden Männern lassen selbstverständlich keine Rückschlüsse über Zusammenhänge auf der Individualebene zu. 
nern in MV sind egalitäre Vorstellungen von Geschlechterrollen am stärksten verbreitet und sie sehen auch am ehesten positive Konsequenzen der Erwerbstätigkeit von Müttern für die Kinder. Bei Männern in BY und auch in SH sind diese Ansichten deutlich seltener verbreitet. Dies deutet auf nach wie vor existierende Ost-/Westunterschiede in den Einstellungen hin, die sich sowohl auf unterschiedliche Traditionen der Müttererwerbstätigkeit als auch auf variierende Opportunitätsstrukturen und ökonomische Notwendigkeiten der gleichzeitigen Verbindung von Familie und Beruf gründen (Bauernschuster/Rainer 2012; Goerres/Tepe 2012; Lück 2009). Ausgehend davon, dass Männer in BY und SH den Auswirkungen der Erwerbstätigkeit von Müttern auf kleine Kinder mehrheitlich skeptisch gegenüberstehen ${ }^{15}$, ist es zumindest eine realistische Annahme, dass sie den Großteil ihrer Elternzeit auch deshalb ihren Partnerinnen überlassen.

\section{Diskussion und Ausblick}

Die vorliegenden Ergebnisse machen zunächst deutlich, dass Väter, die Elterngeld beziehen und insbesondere solche, die in diesem Zusammenhang ihre Erwerbsbeteiligung reduzieren oder unterbrechen, noch immer eine deutliche Minderheit sind. Die seitens der Politik immer wieder als Erfolg dargestellte zunehmende Väterbeteiligung am Elterngeld kann nicht darüber hinwegtäuschen, dass es ausschließlich die sogenannten Bonusmonate sind, die sich einer im Zeitverlauf wachsenden Beliebtheit erfreuen. Die für die drei exemplarisch ausgewählten Bundesländer ermittelten Ergebnisse verdeutlichen einen bundesweiten Trend, den Rückgang der durchschnittlichen Bezugsdauer des Elterngeldes durch Väter, der vor allem in Bundesländern mit einer überdurchschnittlich hohen Väterbeteiligung zu verzeichnen ist (Statistisches Bundesamt 2012b). Unter Gleichstellungsgesichtspunkten ist dieser Befund bedenklich und fordert zu einem Nachdenken über eine mögliche Ausweitung der Partnermonate heraus (Schwentker 2012).

Darüber hinaus wird der Blick dafür geschärft, dass die Entscheidung des Partners überhaupt Elterngeld zu beziehen oder dies für einen längeren Zeitraum zu tun, tendenziell unterschiedlichen Einflussfaktoren unterliegt. Die Entscheidung für einen Partnerantrag folgt im Wesentlichen familienökonomisch oder verhandlungstheoretisch abgeleiteten Erwartungen. Eine Erwerbstätigkeit und ein hohes Einkommen der Frau erhöhen die Wahrscheinlichkeit eines Elterngeldbezuges des Partners. Im Einklang damit steht auch, dass vor der Geburt des Kindes nicht erwerbstätige Männer deutlich länger Elterngeld beziehen als erwerbstätige Männer. Werden die Analysen jedoch auf die Paare konzentriert, bei denen ein Elterngeldbezug des Mannes auch eine Reduzierung seiner Erwerbsbeteiligung nach sich zieht, so zeigen sich hinsichtlich der Länge der Elternzeit interessante Ergebnisse, die über ausschließlich ökonomisch basierte Erklärungen hinausweisen. So fördern beispielsweise ein nichteheliches Zusammenleben oder das Leben in einer Großstadt

15 In BY stimmten im betrachteten Zeitraum 82 Prozent der Männer der Aussage zu, dass ein Kleinkind leidet, wenn seine Mutter erwerbstätig ist. In SH waren dies 70 Prozent und in MV lediglich 38 Prozent. International vergleichende Analysen dieses Items weisen interessanterweise darauf hin, dass Männer und insbesondere Väter in beiden Teilen Deutschlands deutlich häufiger diese Einschätzung teilen als Frauen bzw. Mütter (Schneider/Becker 2012: 257). 
eine längere Elternzeit des Partners - Befunde, die vermutlich egalitäre Orientierungen im Hinblick auf Geschlechterrollen widerspiegeln. Bislang wenig beachtet ist auch, dass eine höhere Anzahl von Kindern im Haushalt eine längere Elternzeit des Vaters unterstützt. Für ein umfassendes Verständnis dieses Zusammenhangs wäre es wichtig mittels Längsschnittdaten zu ermitteln, welche Rolle bisherige Erfahrungen mit Kindern in der Familie für diesbezügliche Entscheidungen der Eltern spielen.

Insbesondere hinsichtlich des Einflusses ökonomischer Ressourcen auf die Dauer der Elternzeit des Vaters unterstreichen die vorliegenden Ergebnisse die Relevanz des Paarkontextes. Hier zeigt sich einerseits, dass Paare nicht nur Wirtschafts-, sondern auch Solidargemeinschaften sind. Dies kommt darin zum Ausdruck, dass eine längere Elternzeit des Mannes mitunter dazu dient, die spezifische Lebenssituation der Partnerin, beispielsweise die Beendigung einer Ausbildung oder die mit einer Selbstständigkeit verbundenen Unwägbarkeiten, abzusichern. Andererseits wird jedoch auch deutlich, dass gerade eine abhängige Erwerbstätigkeit der Frau eine nur kurzzeitige Elternzeit des Partners begünstigt. Gleiches gilt für ein anteilig mittleres oder hohes Einkommen oder für ein hohes Gesamteinkommen des Paares. Es sind also sehr spezifische Paarkonstellationen, innerhalb derer sich vor der Geburt des Kindes erwerbstätige Männer eine längere berufliche Auszeit nehmen. Dies mag zum einen an den vielfach beschriebenen beruflichen Opportunitätskosten liegen, die sich auch darin ausdrücken, dass eine längere Einschränkung der Erwerbsbeteiligung bei Männern im Arbeitsumfeld besonders legitimationsbedürftig ist (Murgia/Poggio 2013; Richter 2012). Zum anderen scheint sich aber gerade bei vor der Geburt des Kindes erwerbstätigen Paaren, die sich für einen Partnerantrag entscheiden, eine ungeschriebene „12+2-Norm“ etabliert zu haben. In gewisser Weise deutet sich ein „gatekeeping“ der Mütter an, welches es ihnen erschwert, den Partnern einen Teil ,ihrer“ Elternzeit zu überlassen. Insofern würde eine Ausweitung der Partnermonate die Verhandlungsposition von Männern sowohl gegenüber ihren Arbeitgebern als auch gegenüber ihren Partnerinnen verbessern. Falls sich eine derartige Maßnahme weiterhin im Rahmen von 14 Monaten bewegen würde, ist auch damit zu rechnen, dass sie insbesondere bei einem Teil der Frauen auf Unverständnis stößt.

Die Frage, ob das Elterngeld eine katalysatorische Wirkung auf die Gleichstellung der Geschlechter hat, ist derzeit noch schwer zu beantworten. Die sehr selektive insbesondere längerfristige Nutzung durch Väter dämpft diesbezügliche Erwartungen. Nach den Ergebnissen einer 2012 durchgeführten Studie besteht bezüglich der Dauer der Elternzeit, die von Vätern genutzt werden kann, immer noch ein Informationsdefizit (Väter gGmbH 2012: 48), das es zu beseitigen gilt. Erste Untersuchungsbefunde zu Auswirkungen der väterlichen Elternzeit entlang verschiedener Dimensionen geben Anlass zu vorsichtigem Optimismus. Schober (2012) berichtet, dass das zeitliche Engagement der Väter für die Kinderbetreuung seit der Elterngeldreform zugenommen hat und führt dies auf eine Veränderung normativer Erwartungen zurück. Brandth und Kvande (2009) können eine solche Tendenz auch für Norwegen bestätigen. Schulz und Rost (2012) kommen in ihrer bayerischen Fallstudie zu dem Ergebnis, dass Paare, die im Jahr 2007 ein erstes Kind bekamen, dann eine stärker partnerschaftliche Arbeitsteilung im Haushalt praktizierten, wenn die Frauen ihre Erwerbstätigkeit für maximal ein Jahr unterbrachen. Kotsadam und Finseraas (2011) wiederum belegen, dass die Einführung einer vierwöchigen Väterzeit in Norwegen einen langfristigen Effekt auf Konfliktlösungen über die Aufteilung der Hausarbeit und auf Vorstellungen von 
Geschlechterrollen hatte. Eine weitere Entwicklung hin zu einer stärker paritätischen Nutzung von Elterngeld und Elternzeit in Deutschland wird vom Abbau wahrgenommener Hindernisse auf verschiedenen gesellschaftlichen Ebenen abhängen: innerhalb der Familienund Sozialpolitik durch einen kohärenteren Fokus auf Geschlechtergleichstellung, innerhalb der Unternehmen durch eine größere Akzeptanz väterlicher Auszeiten und innerhalb der Paare selbst durch die Bereitschaft die Verbindung von Familie und Beruf als partnerschaftliche Aufgabe aufzufassen. Eine systematische Untersuchung der Langzeitwirkungen einer stärkeren Beteiligung von Vätern an Elterngeld und Elternzeit im Hinblick auf die Aufteilung von Erwerbs- und Familienarbeit innerhalb der Partnerschaft steht ebenso noch aus wie bezüglich der Vater-Kind-Beziehung (BMFSFJ 2011).

\section{Literatur}

Auspurg, K. \& Hinz, T. (2011). Gruppenvergleiche bei Regressionen mit binären abhängigen Variablen - Probleme und Fehleinschätzungen am Beispiel von Bildungschancen im Kohortenverlauf. Zeitschrift für Soziologie, 40, 1, S. 62-73.

Baronsky, A., Gerlach, I. \& Schneider, A. K. (2012). Väter in der Familienpolitik. Aus Politik und Zeitgeschichte, 62, 40, S. 31-36.

Bauernschuster, S. \& Rainer, H. (2012). Political regimes and the family: How sex-role attitudes continue to differ in reunified Germany. Journal of Population Economics, 25, 1, S. 5-27.

Beblo, M. (2012). Der Einfluss institutioneller Rahmenbedingungen auf die Arbeitsteilung von Paaren. In: Bertram. H. \& Bujard, M. (Hrsg.), Zeit, Geld, Infrastruktur - zur Zukunft der Familienpolitik. Soziale Welt - Sonderband 19. Baden-Baden: Nomos, 193-207.

Becker, G. S. (1991). A treatise on the family. Cambridge, MA: Harvard University Press.

Berk, R.A. \& Berk, S.F. (1983). Supply-side sociology of the family: The challenge of the new home economics. Annual Review of Sociology, 9, S. 375-395.

Best, H. \& Wolf, C. (2010). Logistische Regression. In: Wolf, C. \& Best, H. (Hrsg.). Handbuch der sozialwissenschaftlichen Datenanalyse. Wiesbaden: VS Verlag für Sozialwissenschaften, S. 827-854.

Best, H. \& Wolf, C. (2012). Modellvergleich und Ergebnisinterpretation in Logit- und Probit-Regressionen. Kölner Zeitschrift für Soziologie und Sozialpsychologie, 64, 2, S. 377-395.

Blood, R.O. \& Wolfe, D.M. (1960). Husbands and wives. The dynamics of married living. Glencoe, IL: The Free Press.

BMFSFJ (2011). Vaterschaft und Elternzeit. Berlin: BMFSFJ. www.bmfsfj.de/Redaktion BMFSFJ/ Broschuerenstelle/Pdf-Anlagen/vaterschaft-und-elternzeit-endbericht,property=pdf,bereich=bmfsfj, sprache $=$ de, $r w b=$ true.pdf (Stand: 2013-02-21).

BMFSFJ (2012a). Das Elterngeld ist ein Erfolgsmodell, Berlin: Pressemitteilung des BMFSFJ vom 27.02.2012. www.bmfsfj.de/BMFSFJ/Presse/pressemitteilungen,did=184506.html (Stand: 2013-0127).

BMFSFJ (2012b). Vom Elterngeld profitieren Mütter, immer mehr Väter - und vor allem die Kinder, Berlin: BMFSFJ (Pressemitteilung des BMFSFJ vom 27.06.2012). www.bmfsfj.de/BMFSFJ/Presse/ pressemitteilungen,did=187190.html (Stand: 2013-01-27).

BMFSFJ (2012c). Elterngeld und Elternzeit - Das Bundeselterngeld- und Elternzeitgesetz, Berlin: BMFSFJ. www.bmfsfj.de/BMFSFJ/familie,did=89272.html (Stand: 2013-02-08).

Brandth, B. \& Kvande, E. (2009). Gendered or gender-neutral care politics for fathers? The ANNALS of the American Academy of Political and Social Science, 624, 1, S. 177-189.

Bundesagentur für Arbeit (2010). Arbeitsmarkt 2010. Amtliche Nachrichten der Bundesagentur für Statistik.statistik.arbeitsagentur.de/Statischer-Content/Arbeitsmarktberichte/JahresberichtArbeitsmarkt-Deutschland/Generische-Publikationen/Arbeitsmarkt-2010.pdf (Stand: 2013-02-06). 
Bygren, M. \& Duvander, A.-Z. (2006). Parents' workplace situation and fathers' parental leave use. Journal of Marriage and Family, 68, 2, S. 363-372.

Coltrane, S. (2009). Fatherhood, gender and work-family policies. In: Gornick, J. C. \& Meyers, M. K. (Hrsg.), Gender equality: Transforming family divisions of labor. London \& New York: Verso, S. 385-409

Duvander, A.-Z. \& Johansson, M. (2012). What are the effects of reforms promoting fathers' parental leave use? Journal of European Social Policy, 22, 3, S. 319-330.

Geisler, E. \& Kreyenfeld, M. (2011). Against all odds: Fathers' use of parental leave in Germany. Journal of European Social Policy, 21, 1, S. 88-99.

Geisler, E. \& Kreyenfeld, M. (2012). How policy matters: Germany's parental leave benefit reform and fathers' behavior 1999-2009. Rostock: Max Planck Institute for Demographic Research (MPIDR Working Paper 2012-021).

Goerres, A. \& Tepe, M. (2012). Doing it for the kids? The determinants of attitudes towards public childcare in unified Germany. Journal of Social Policy, 41, 2, S. 349-372.

Haas, L. \& Rostgaard, T. (2011). Fathers' rights to paid parental leave in the Nordic countries: Consequences for the gendered division of leave. Community, Work and Family, 14, 2, S. 177-195.

Initiative Neue Soziale Marktwirtschaft (2012). Bundesländerranking 2012. www.bundeslaenderranking. de (Stand: 2013-02-06).

Kotsadam, A. \& Finseraas, H. (2011). The state intervenes in the battle of the sexes: Causal effects of paternity leave. Social Science Research, 40, 6, S. 1611-1622.

Lappegard, T. (2008). Changing the gender balance in caring: Fatherhood and the division of parental leave in Norway. Population Research and Policy Review, 27, 2, S. 139-159.

Lück, D. (2009). Der zögernde Abschied vom Patriarchat: Der Wandel von Geschlechterrollen im internationalen Vergleich. Berlin: edition sigma.

Lundberg, S. \& Pollak, R. A. (1996). Bargaining and distribution in marriage. Journal of Economic Perspectives, 10 , 4, S. 139-158.

Mannino, C. \& Deutsch, F. (2007). Changing the division of household labor: A negotiated process between partners. Sex Roles, 56, 5-6, S. 309-324.

Mood, C. (2010). Logistic regression: Why we cannot do what we think we can do, and what we can do about it. European Sociological Review, 26, 1, S. 67-82.

Murgia, A. \& Poggio, B. (2013). Fathers' stories of resistance and hegemony in organizational cultures. Gender, Work and Organization, 20, 4, S. 413-424.

Naz, G. (2010). Usage of parental leave by fathers in Norway. International Journal of Sociology and Social Policy, 30, 5-6, S. 313-325.

Ott, N. (1992). Intrafamily bargaining and household decisions. Berlin, Heidelberg \& New York: Springer.

Pfahl, S. \& Reuyß, S. (2010). Das neue Elterngeld: Erfahrungen und betriebliche Nutzungsbedingungen von Vätern. In: Badura, B., Schröder, H., Klose, J. \& Macco, K. (Hrsg.), Fehlzeiten-Report 2010. Berlin, Heidelberg \& New York: Springer, S. 225-233.

Pull, K. \& Vogt, A. C. (2010). Viel Lärm um Nichts? Soziale Welt, 61, 2, S. 121-137.

Reich, N. (2011). Predictors of fathers' use of parental leave in Germany. Population Review, 50, 2, S. 1-22.

Rheinisch-Westfälisches Institut für Wirtschaftsforschung (RWI) (2008). Evaluation des Gesetzes zum Elterngeld und zur Elternzeit, Endbericht. Studie im Auftrag des BMFSFJ. Essen: Rheinisch-Westfälisches Institut für Wirtschaftsforschung.

Rheinisch-Westfälisches Institut für Wirtschaftsforschung (RWI) (2009). Evaluationsbericht Bundeselterngeld- und Elternzeitgesetz 2009. Studie im Auftrag des BMFSFJ. Essen: Rheinisch-Westfälisches Institut für Wirtschaftsforschung.

Richter, R. (2012). Väter in Elternzeit: Umsetzungen und Strategien zwischen Familie und Beruf. Paderborn: Universität Paderborn (Dissertation). digital.ub.uni-paderborn.de/ubpb/urn/urn:nbn:de:hbz: 466:2-9359 (Stand: 2013-02-04).

Schneider, N. F. (2010). Das veränderte Selbstverständnis von Eltern heute und die veränderte Rolle des Kindes. In: Henry-Huthmacher, C. \& Hoffmann, E. (Hrsg.). Wenn Eltern nur das Beste wollen...: 
Ergebnisse einer Expertenrunde der Konrad-Adenauer-Stiftung. Sankt Augustin: Konrad-AdenauerStiftung, S. 13-15.

Schneider, N. F. \& Becker, K. S. (2012). Fatherhood in times of gender transformation - European perspectives. In: Oechsle, M., Müller, U. \& Hess, S. (Hrsg.). Fatherhood in late modernity: Cultural images, social practices, structural frames. Opladen, Berlin \& Toronto: Barbara Budrich, S. 249-271.

Schober, P. S. (2012). Parental leave policies and child care time in couples after childbirth. Berlin: Deutsches Institut für Wirtschaftsforschung (SOEPaper 434).

Schulz, F. \& Rost, H. (2012). Hausarbeitsteilung und Erwerbsunterbrechung von Müttern unter den Bedingungen des neuen Elterngeldgesetzes. Erste empirische Befunde aus Bayern. Zeitschrift für Familienforschung/Journal of Family Research, 24, 1, S. 27-45.

Schwentker, B. (2012). Land ohne Väter. Demografie-Blog. www.demografie-blog.de/2012/06/landohne-vater (Stand: 2013-02-20).

Statistisches Bundesamt (2012a). Pressekonferenz ,Elterngeld - wer, wie lange und wie viel? am 27. Juni 2012 in Berlin, Statement von Präsident Roderich Egeler. www.destatis.de/DE/Presse Service/Presse/Pressekonferenzen/2012/Elterngeld/statement_egeler_elterngeld_PDF.pdf?_blob= publicationFile (Stand: 2013-01-27).

Statistisches Bundesamt (2012b). Pressekonferenz „Elterngeld - wer, wie lange und wie viel? am 27. Juni 2012 in Berlin, Begleitmaterial zur Pressekonferenz. www.destatis.de/DE/PresseService/Presse/ Pressekonferenzen/2012/Elterngeld/begleitmaterial_PDF.pdf?_blob=publicationFile $<01-02-2013>$.

Statistisches Bundesamt (2013). Elterngeld: Väterbeteiligung mit 27,3\% auf neuem Höchststand, Pressemitteilung vom 27. Mai 2013. www.destatis.de/DE/PresseService/Presse/Pressemitteilungen/ 2013/05/PD13_176_22922pdf.pdf?_blob=publicationFile $<29-06-2013>$.

Väter gGmbH (2012). Trendstudie „Moderne Väter": Wie die neue Vätergeneration Familie, Gesellschaft und Wirtschaft verändert. Hamburg: Väter gGmbH.

Sundström, M. \& Duvander, A.-Z. (2002). Gender division of childcare and the sharing of parental leave among new parents in Sweden. European Sociological Review, 18, 4, S. 433-447.

Trappe, H. (2013). Väter mit Elterngeldbezug: Nichts als ökonomisches Kalkül? Zeitschrift für Soziologie $42,1, \mathrm{~S} .28-51$.

West, C. \& Zimmerman, D. H. (1987). Doing gender. Gender and Society, 1, 2, S. 125-151.

Eingereicht am/Submitted on: 10.04.2013

Angenommen am/Accepted on: 02.07.2013

Anschrift der Autorin/Address of the author:

Prof. Dr. Heike Trappe

Universität Rostock

Wirtschafts- und Sozialwissenschaftliche Fakultät

Institut für Soziologie und Demographie

Ulmenstraße 69

18057 Rostock

Deutschland/Germany

E-Mail: heike.trappe@uni-rostock.de

\section{Anhang}


Tabelle A1: Determinanten eines Partnerantrags - Beschreibung des Samples

\begin{tabular}{|c|c|c|c|c|}
\hline & \multicolumn{4}{|c|}{ Elterngeldanträge in } \\
\hline & $\begin{array}{l}\text { Alle Eltern- } \\
\text { geldanträge }\end{array}$ & Bayern (BY) & $\begin{array}{c}\text { Mecklenburg- } \\
\text { Vorpommern (MV) }\end{array}$ & $\begin{array}{c}\text { Schleswig-Holstein } \\
\text { (SH) }\end{array}$ \\
\hline \multicolumn{5}{|c|}{ Partnerantrag } \\
\hline $\mathrm{Ja}$ & 24,7 & 26,7 & 19,2 & 18,0 \\
\hline Nein & 75,3 & 73,3 & 80,8 & 82,0 \\
\hline \multicolumn{5}{|c|}{ Jahr der Geburt des Kindes } \\
\hline 2007 & 33,7 & 33,8 & 33,0 & 34,0 \\
\hline 2008 & 33,6 & 33,6 & 33,7 & 33,6 \\
\hline 2009 & 32,7 & 32,6 & 33,3 & 32,4 \\
\hline
\end{tabular}

Bundesland

$\begin{array}{lr}\text { BY } & 74,8 \\ \text { MV } & 9,2\end{array}$

SH 16,0

\begin{tabular}{ll} 
Wohnort & \\
keine Großstadt & 75,5 \\
München & 13,0 \\
andere Großstadt & 11,5 \\
\hline
\end{tabular}

\begin{tabular}{|c|c|c|c|c|}
\hline \multicolumn{5}{|l|}{ Familienstand der Frau } \\
\hline verheiratet & 72,6 & 76,1 & 48,4 & 69,8 \\
\hline eingetr. Lebenspartnerschaft & 0,3 & 0,3 & 0,1 & 0,1 \\
\hline Ledig & 22,9 & 18,5 & 48,9 & 29,2 \\
\hline verwitwet/geschieden & 1,9 & 2,0 & 2,5 & 0,8 \\
\hline keine Angabe & 2,3 & 3,1 & 0,1 & 0,1 \\
\hline \multicolumn{5}{|l|}{ Anzahl der Kinder im Haushalt } \\
\hline 1 Kind & 51,9 & 51,7 & 53,5 & 52,0 \\
\hline 2 Kinder & 33,9 & 34,2 & 33,4 & 33,0 \\
\hline 3 und mehr Kinder & 14,2 & 14,1 & 13,1 & 15,0 \\
\hline \multicolumn{5}{|l|}{ Altersgruppe der Frau } \\
\hline unter 30 Jahre & 37,4 & 33,6 & 58,7 & 43,2 \\
\hline 30 bis 34 Jahre & 33,4 & 34,6 & 26,5 & 31,4 \\
\hline 35 Jahre und älter & 29,2 & 31,8 & 14,8 & 25,4 \\
\hline \multicolumn{5}{|c|}{ Erwerbsstatus der Frau ein Jahr vor der Geburt } \\
\hline abhängige Erwerbstätigkeit & 65,2 & 67,0 & 55,3 & 62,5 \\
\hline selbstständig & 2,6 & 2,5 & 2,6 & 2,8 \\
\hline in Ausbildung & 1,7 & 0,9 & 6,4 & 2,6 \\
\hline Hausfrau & 24,5 & 25,4 & 10,8 & 28,4 \\
\hline arbeitslos & 6,0 & 4,2 & 24,9 & 3,7 \\
\hline \multicolumn{5}{|c|}{ Erwerbseinkommen der Frau ein Jahr vor der Geburt } \\
\hline Keines & 32,4 & 30,7 & 42,2 & 34,8 \\
\hline bis zu 1.000 Euro & 32,7 & 32,6 & 31,9 & 33,3 \\
\hline 1.001-2.000 Euro & 27,2 & 28,1 & 22,8 & 25,8 \\
\hline mehr als 2.000 Euro & 7,7 & 8,6 & 3,1 & 6,1 \\
\hline Fallzahl & 411.088 & 307.525 & 37.832 & 65.731 \\
\hline
\end{tabular}

Quelle: Daten des Elterngeldbezugs in BY bereitgestellt durch das Zentrum Bayern Familie und Soziales, Daten des Elterngeldbezugs in MV bereitgestellt durch das Landesamt für Gesundheit und Soziales (LAGuS), Daten des Elterngeldbezugs in SH bereitgestellt durch das Landesamt für soziale Dienste, eigene Berechnungen. 
Tabelle A2: Determinanten der Aufteilung des Elterngeldbezugs (Paare mit Partnerantrag) - Beschreibung des Samples

\begin{tabular}{|c|c|c|c|c|c|c|c|c|}
\hline & \multicolumn{2}{|c|}{ Alle Paare } & \multicolumn{2}{|c|}{ Paare in BY } & \multicolumn{2}{|c|}{ Paare in MV } & \multicolumn{2}{|c|}{ Paare in SH } \\
\hline & $\begin{array}{l}\text { Mann } \\
\text { erwerbs- } \\
\text { tätig }\end{array}$ & $\begin{array}{l}\text { Doppel- } \\
\text { verdiener- } \\
\text { paar }\end{array}$ & $\begin{array}{l}\text { Mann } \\
\text { erwerbs- } \\
\text { tätig }\end{array}$ & $\begin{array}{l}\text { Doppel- } \\
\text { verdiener- } \\
\text { paar }\end{array}$ & $\begin{array}{l}\text { Mann } \\
\text { erwerbs- } \\
\text { tätig }\end{array}$ & $\begin{array}{l}\text { Doppel- } \\
\text { verdiener- } \\
\text { paar }\end{array}$ & $\begin{array}{l}\text { Mann } \\
\text { erwerbs- } \\
\text { tätig }\end{array}$ & $\begin{array}{l}\text { Doppel- } \\
\text { verdiener- } \\
\text { paar }\end{array}$ \\
\hline \multicolumn{9}{|c|}{ Dauer des Elterngeldbezugs durch Partner } \\
\hline 1-2 Monate & 81,4 & 83,5 & 82,3 & 84,4 & 77,3 & 79,4 & 77,4 & 79,2 \\
\hline mehr als $2 \mathrm{M}$. & 18,6 & 16,5 & 17,7 & 15,6 & 22,7 & 20,6 & 22,6 & 20,8 \\
\hline \multicolumn{9}{|c|}{ Jahr der Geburt des Kindes } \\
\hline 2007 & 27,4 & 27,1 & 27,0 & 26,7 & 29,6 & 29,4 & 29,2 & 28,8 \\
\hline 2008 & 34,8 & 34,7 & 34,6 & 34,5 & 36,6 & 36,7 & 34,7 & 34,7 \\
\hline 2009 & 37,8 & 38,2 & 38,4 & 38,8 & 33,8 & 33,9 & 36,1 & 36,5 \\
\hline \multicolumn{9}{|l|}{ Bundesland } \\
\hline BY & 82,9 & 82,4 & & & & & & \\
\hline MV & 6,2 & 6,3 & & & & & & \\
\hline $\mathrm{SH}$ & 10,9 & 11,3 & & & & & & \\
\hline \multicolumn{9}{|l|}{ Wohnort } \\
\hline keine Großstadt & & & 76,8 & 76,7 & & & & \\
\hline München & & & 13,4 & 13,8 & & & & \\
\hline andere Großstadt & & & 9,8 & 9,5 & & & & \\
\hline \multicolumn{9}{|c|}{ Partnerschaftsform } \\
\hline verheiratet & 79,6 & 78,3 & 81,1 & 79,7 & 62,9 & 63,3 & 77,3 & 76,6 \\
\hline NEL & 20,4 & 21,7 & 18,9 & 20,3 & 37,1 & 36,7 & 22,7 & 23,4 \\
\hline \multicolumn{9}{|c|}{ Anzahl der Kinder im Haushalt } \\
\hline $1 \mathrm{Kind}$ & 57,1 & 62,5 & 56,8 & 62,9 & 55,5 & 56,1 & 60,1 & 63,7 \\
\hline 2 Kinder & 32,2 & 29,6 & 32,3 & 29,2 & 34,8 & 35,3 & 30,2 & 28,6 \\
\hline $3+$ Kinder & 10,7 & 7,9 & 10,9 & 7,9 & 9,7 & 8,6 & 9,7 & 7,7 \\
\hline \multicolumn{9}{|c|}{ Altersgruppe der Frau } \\
\hline unter 30 Jahre & 24,1 & 22,7 & 22,5 & 21,3 & 41,0 & 37,8 & 26,9 & 24,3 \\
\hline 30 - 34 Jahre & 39,8 & 40,9 & 40,0 & 40,9 & 37,8 & 40,0 & 39,9 & 41,5 \\
\hline 35 + Jahre & 36,1 & 36,4 & 37,5 & 37,8 & 21,2 & 22,2 & 33,2 & 34,2 \\
\hline \multicolumn{9}{|c|}{ Altersgruppe des Mannes } \\
\hline unter 30 Jahre & 10,8 & 10,3 & 9,8 & 9,4 & 21,6 & 19,5 & 12,8 & 11,6 \\
\hline 30 - 34 Jahre & 29,7 & 30,4 & 29,2 & 29,9 & 35,3 & 36,4 & 30,6 & 30,9 \\
\hline $35+$ Jahre & 59,5 & 59,3 & 61,0 & 60,7 & 43,1 & 44,1 & 56,6 & 57,5 \\
\hline \multicolumn{9}{|c|}{ Altersdifferenz der Partner } \\
\hline M./F. $\geq 3 \mathrm{~J}$ & 44,0 & 44,9 & 44,0 & 44,9 & 45,3 & 46,7 & 43,3 & 43,9 \\
\hline M./F. > $4 \mathrm{~J}$. & 40,7 & 39,4 & 40,7 & 39,5 & 39,3 & 37,6 & 41,2 & 40,2 \\
\hline$M$. jünger $F$. & 15,3 & 15,7 & 15,3 & 15,6 & 15,4 & 15,7 & 15,5 & 15,9 \\
\hline \multicolumn{9}{|c|}{ Erwerbsstatus der Frau ein Jahr vor der Geburt } \\
\hline \multicolumn{9}{|c|}{ Abhängig } \\
\hline beschäftigt & 79,8 & 95,1 & 79,5 & 95,3 & 80,6 & 93,4 & 82,1 & 94,1 \\
\hline selbstständig & 4,2 & 4,9 & 3,9 & 4,7 & 5,7 & 6,6 & 5,1 & 5,9 \\
\hline in Ausbildung & 0,9 & - & 0,6 & - & 4,2 & - & 1,8 & - \\
\hline Hausfrau & 13,7 & - & 15,0 & - & 3,7 & - & 9,7 & - \\
\hline arbeitslos & 1,4 & - & 1,0 & - & 5,8 & - & 1,3 & - \\
\hline
\end{tabular}




\begin{tabular}{|c|c|c|c|c|c|c|c|c|}
\hline & & & & & & & & \\
\hline & $\begin{array}{l}\text { Mann } \\
\text { erwerbs- } \\
\text { tätig }\end{array}$ & $\begin{array}{l}\text { Doppel- } \\
\text { verdiener- } \\
\text { paar }\end{array}$ & $\begin{array}{l}\text { Mann } \\
\text { erwerbs- } \\
\text { tätig }\end{array}$ & $\begin{array}{l}\text { Doppel- } \\
\text { verdiener- } \\
\text { paar }\end{array}$ & $\begin{array}{l}\text { Mann } \\
\text { erwerbs- } \\
\text { tätig }\end{array}$ & $\begin{array}{l}\text { Doppel- } \\
\text { verdiener- } \\
\text { paar }\end{array}$ & $\begin{array}{l}\text { Mann } \\
\text { erwerbs- } \\
\text { tätig }\end{array}$ & $\begin{array}{l}\text { Doppel- } \\
\text { verdiener- } \\
\text { paar }\end{array}$ \\
\hline Erwerbsstatus & s Mannes & in Jahr vor d & er Geburt & & & & & \\
\hline Abhängig & & & & & & & & \\
\hline beschäftigt & 87,6 & 87,4 & 88,2 & 87,9 & 84,2 & 84,4 & 85,5 & 85,5 \\
\hline selbstständig & 12,4 & 12,6 & 11,8 & 12,1 & 15,8 & 15,6 & 14,5 & 14,5 \\
\hline $\begin{array}{l}\text { Erwerbsstatus } \\
\text { beide }\end{array}$ & ider Partne & r ein Jahr vo & der Gebur & & & & & \\
\hline $\begin{array}{l}\text { erwerbstätig } \\
\text { nur Mann }\end{array}$ & 84,0 & & 83,4 & & 86,3 & & 87,2 & \\
\hline erwerbstätig & 16,0 & & 16,6 & & 13,7 & & 12,8 & \\
\hline Erwerbseinkor & en der Fra & ein Jahr vor & der Geburt & & & & & \\
\hline keines & 16,7 & - & 17,3 & - & 14,0 & - & 13,0 & - \\
\hline bis $z u \in 1.000$ & 28,2 & 34,3 & 28,2 & 34,7 & 30,3 & 35,5 & 27,0 & 31,2 \\
\hline$€ 1.001-2.000$ & 39,9 & 47,6 & 39,0 & 46,7 & 47,2 & 54,7 & 43,4 & 49,7 \\
\hline$€ 2.000+$ & 15,2 & 18,1 & 15,5 & 18,6 & 8,5 & 9,8 & 16,6 & 19,1 \\
\hline Erwerbseinkor & ien des Ma & nes ein Jahr & vor der $\mathrm{Ge}$ & ourt & & & & \\
\hline bis $z u € 1.000$ & 15,3 & 14,8 & 13,7 & 13,5 & 29,7 & 27,7 & 18,5 & 17,2 \\
\hline$€ 1.001-2.000$ & 42,7 & 44,1 & 42,0 & 43,4 & 52,1 & 53,7 & 42,8 & 44,0 \\
\hline$€ 2.000+$ & 42,0 & 41,1 & 44,3 & 43,1 & 18,2 & 18,6 & 38,7 & 38,8 \\
\hline Anteil des Erw & seinkomm & ens der Frau & am Erwerbs & einkommen & des Partner & & & \\
\hline$<50 \%$ & 39,5 & 28,0 & 41,1 & 29,4 & 25,8 & 14,1 & 35,0 & 25,5 \\
\hline $50-79 \%$ & 19,2 & 22,9 & 19,3 & 23,2 & 19,3 & 22,3 & 18,4 & 21,1 \\
\hline $80-119 \%$ & 25,1 & 29,8 & 24,4 & 29,2 & 31,2 & 36,2 & 26,9 & 30,8 \\
\hline$\geq 120 \%$ & 16,2 & 19,3 & 15,2 & 18,2 & 23,7 & 27,4 & 19,7 & 22,6 \\
\hline Summe der En & rbseinkom & nen beider $P$ & artner & & & & & \\
\hline bis $z u € 2.000$ & 17,6 & 10,9 & 16,6 & 10,0 & 30,2 & 21,6 & 18,0 & 11,6 \\
\hline$€ 2.001-3.000$ & 35,5 & 33,4 & 35,5 & 32,8 & 40,2 & 44,5 & 32,4 & 31,7 \\
\hline$€ 3.001-4.000$ & 29,8 & 35,3 & 30,1 & 35,9 & 20,7 & 23,7 & 32,8 & 37,5 \\
\hline$€ 4.000+$ & 17,1 & 20,4 & 17,8 & 21,3 & 8,9 & 10,2 & 16,8 & 19,2 \\
\hline Fallzahl & 90.755 & 76.220 & 75.279 & 62.776 & 5.593 & 4.827 & 9.883 & 8.617 \\
\hline
\end{tabular}

Quelle: Daten des Elterngeldbezugs in BY bereitgestellt durch das Zentrum Bayern Familie und Soziales, Daten des Elterngeldbezugs in MV bereitgestellt durch das Landesamt für Gesundheit und Soziales (LAGuS), Daten des Elterngeldbezugs in SH bereitgestellt durch das Landesamt für soziale Dienste, eigene Berechnungen. 


\section{Eltern Stärken - \\ jetzt in 4., überarbeiteter Auflage}

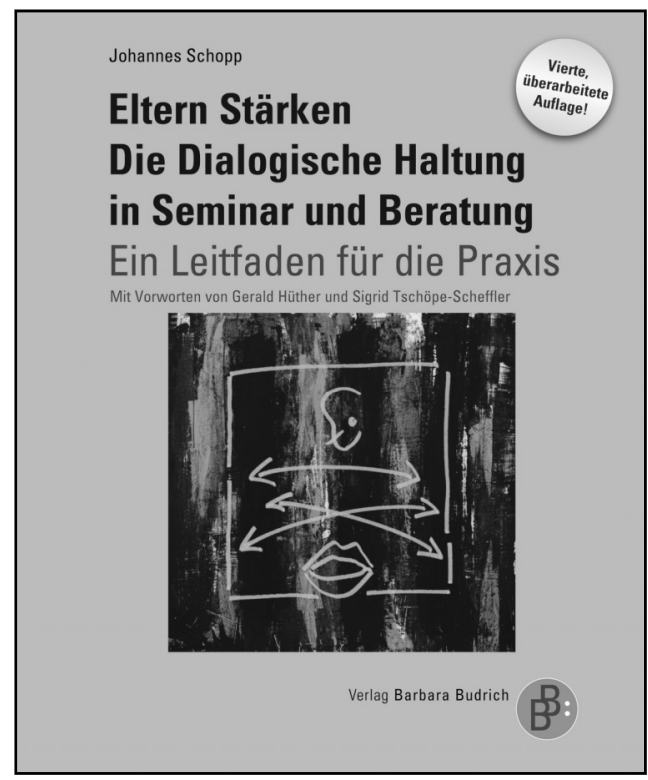

Johannes Schopp

Eltern Stärken

Die Dialogische Haltung in Seminar und Beratung

Ein Leitfaden für die Praxis

4., überarbeitete Auflage 2013.

283 Seiten. Kart. $19,90 €$

ISBN 978-3-8474-0126-1

Mit Vorworten von Gerald Hüther und Sigrid Tschöpe-Scheffler

Ein Plädoyer für eine Dialogische Kultur, die es schafft, dass Erwachsene untereinander und auch Erwachsene und Kinder sich gleichwürdig begegnen, sich bedingungslos schätzen und respektieren. Eine Kultur, die Bildung als Persönlichkeitsbildung versteht.

Jetzt in Ihrer Buchhandlung bestellen oder direkt bei:

Verlag Barbara Budrich •

\section{Barbara Budrich Publishers}

Stauffenbergstr. 7. D-51379 Leverkusen Opladen

Tel +49 (0)2171.344.594 • Fax +49 (0)2171.344.693•

info@budrich.de

www.budrich-verlag.de 